ARTICLE

https://doi.org/10.1038/s41467-019-12142-4

\title{
Near-infrared light and tumor microenvironment dual responsive size-switchable nanocapsules for multimodal tumor theranostics
}

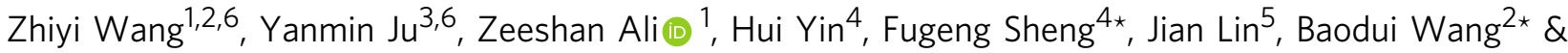 \\ Yanglong Hou (1* ${ }^{1 \star}$
}

Smart drug delivery systems (SDDSs) for cancer treatment are of considerable interest in the field of theranostics. However, developing SDDSs with early diagnostic capability, enhanced drug delivery and efficient biodegradability still remains a scientific challenge. Herein, we report near-infrared light and tumor microenvironment (TME), dual responsive as well as size-switchable nanocapsules. These nanocapsules are made of a PLGA-polymer matrix coated with $\mathrm{Fe} / \mathrm{FeO}$ core-shell nanocrystals and co-loaded with chemotherapy drug and photothermal agent. Smartly engineered nanocapsules can not only shrink and decompose into small-sized nanodrugs upon drug release but also can regulate the TME to overproduce reactive oxygen species for enhanced synergistic therapy in tumors. In vivo experiments demonstrate that these nanocapsules can target to tumor sites through fluorescence/magnetic resonance imaging and offer remarkable therapeutic results. Our synthetic strategy provides a platform for next generation smart nanocapsules with enhanced permeability and retention effect, multimodal anticancer theranostics, and biodegradability.

\footnotetext{
${ }^{1}$ Beijing Key Laboratory for Magnetoelectric Materials and Devices, Department of Materials Science and Engineering, College of Engineering, Beijing Innovation Centre for Engineering Science and Advanced Technology, Peking University, 100871 Beijing, China. ${ }^{2}$ State Key Laboratory of Applied Organic Chemistry, Key Laboratory of Nonferrous Metal Chemistry and Resources Utilization of Gansu Province, Lanzhou University, Gansu 730000 Lanzhou, China. ${ }^{3}$ College of Life Science, Peking University, 100871 Beijing, China. ${ }^{4}$ Department of Radiology, the Fifth Medical Centre, Chinese PLA General Hospital, 100071 Beijing, China. ${ }^{5}$ Synthetic and Functional Biomolecules Center, Department of Chemical Biology, College of Chemistry and Molecular Engineering, Peking University, 100871 Beijing, China. ${ }^{6}$ These authors contributed equally: Zhiyi Wang, Yanmin Ju. *email: fugeng_sheng@163.com; wangbd@lzu.edu.cn; hou@pku.edu.cn
} 
S mart drug delivery systems (SDDSs) have emerged as promising tools for the treatment of malignancies ${ }^{1-4}$. The key features of ideal SDDSs are the enhanced permeability and retention effect (EPR effect) across complex biological systems, "on-demand" drug release capability, and excellent biocompatibility and biodegradatibility ${ }^{5-12}$. As far as nanomedicine is concerned, on-demand change of size of SDDSs in different environments is critical for efficient transportation of nanocarriers to tumor location ${ }^{7,13}$. Generally speaking, nanoparticles (NPs) with a size of 100-200 nm can improve the circulatory halflife, but they are not easy to penetrate deep cellular layers near the tumor vessels ${ }^{1,14,15}$. On the contrary, small size NPs with a diameter of 4-20 nm easily penetrate into deep tumor tissues, but they are more prone to rapid clearance and insufficient drug retention. To tackle these biological barriers, nanocapsules with size switchable function in different biological environments have been developed. With a large initial size of these nanocapsules can achieve effective EPR-derived tumor accumulation during blood circulation, and then these nanocapsules responsively shrink and become small sized once inside the tumor $7,13,16$. However, to the best of our knowledge, reports on such SDDSs are very limited.

Meanwhile, an emerging area of cancer treatment is designing a series of drugs, which can utilize tumor microenvironment (TME) to improve the effect of tumor therapy ${ }^{17-19}$. It is well known that the rapid growth of tumor cells and distortion of tumor blood vessels often results in insufficient oxygen supply and acidification in solid tumors. Hypoxia and acidic $\mathrm{pH}$ in the TME not only accelerate angiogenesis and metastasis of tumors, but also lead to therapeutic resistance and ineffective tumor treatment ${ }^{20-23}$. In particular with regard to photodynamic therapy (PDT) and chemodynamic therapy (CDT), in which oxygen participates in cell killing process, the presence of hypoxia in TME will cause the failure of cancer treatment ${ }^{24-27}$. Recently, various innovative approaches have been explored to overcome the therapeutic resistance caused by hypoxia. Such as, using oxygen "shuttles" (e.g., perfluorocarbon) to deliver oxygen into tumors, or in situ oxygen generation inside the tumor with catalysts, which show promising results in improving the efficacy of PDT and $\mathrm{CDT}^{8-11,24}$. However, there are still many challenges in developing TME-sensitive SDDSs to overcome the anaerobic problem and improve the therapeutic effect.

Moreover, according to the requirements of US Food and Drug Administration (FDA), theranostic agents should be completely cleared from the body within a reasonable period of time. Poly (lactic-co-glycolic) acid (PLGA) is the typical polymer approved by US FDA, which offers several advantages in the design of SDDSs. It has been reported that the nanocarriers formed by thermosensitive PLGA can change shape and release payloads quickly when the system temperature is higher than their phasetransition temperature ${ }^{28}$. Simultaneously, as a clinical diagnostic and near-infrared (NIR) fluorescence imaging agent, indocyanine green (ICG) has been approved by the US FDA. ICG has been studied extensively in PDT and photothermal therapy (PTT) due to its remarkable NIR optical properties in the best light transmission window for biomedical applications ${ }^{29-34}$. Iron-based magnetic nanocrystals (NCs) with magnetic resonance imaging (MRI) are known to have high catalytic activity toward $\mathrm{H}_{2} \mathrm{O}_{2}$ to generate oxidative hydroxyl radicals $(\bullet \mathrm{OH})$ and would decompose under acidic $\mathrm{pH}$, showing great potential applications in $\mathrm{CDT}^{26}$. We hypothesize that the combination of these materials into "all in one" SDDSs may provide for enhanced tumor accumulation and highly effective diagnostics and therapeutics.

Herein, PLGA-polyethylene glycol-poly ( $N$-isopropyl acrylamide), termed as PPP, was synthesized and tethered with $\mathrm{Fe} / \mathrm{FeO}$ $\mathrm{NCs}$ to form Fe/FeO-PPP heterostructures. Doxorubicin (DOX) and ICG were co-loaded into Fe/FeO-PPP heterostructures to develop flexible DOX-ICG@Fe/FeO-PPP nanocapsules by water-oil-water (W/O/W) emulsion method. The resulting nanocapsules, on the one hand, can in situ overproduce reactive oxygen species (ROS) by reacting with endogenous $\mathrm{H}_{2} \mathrm{O}_{2}$ in tumors, which is expected to overcome the tumor hypoxia-related drug resistance of PDT and chemotherapy. On the other hand, DOX-ICG@Fe/FeO-PPP nanocapsules could shrink and decompose into small-sized nanodrugs triggered by photothermal effect of ICG under laser irradiation and lower $\mathrm{pH}$ value in TME. These capabilities show significantly enhanced intratumoral permeability to further improve the therapeutic effect in combination therapy with chemodynamic, photodynamic and chemotherapy. Owing to $\mathrm{Fe} / \mathrm{FeO} \mathrm{NCs}$ as MRI contrast agent and ICG as NIR imaging agent, DOX-ICG@Fe/FeO-PPP-FA nanocapsules can achieve imaging-guided synergetic therapy, which can provide essential information, including tumor size and location, optimal treatment time window and real-time efficacy evaluation.

\section{Results}

Characterization of DOX-ICG@Fe/FeO-PPP nanocapsules. The procedure for the synthesis of DOX-ICG@Fe/FeO-PPP nanocapsules is presented in Fig. 1a. Firstly, monodispersed $\mathrm{Fe} / \mathrm{FeO}$ NCs were synthesized by seed-mediated growth method with thermal decomposition in oil phase. A temperature-responsive multiblock polymer PLGA-PEG-PNIPAM (PPP) was synthesized by covalent bonding subsequently (Fig. $1 \mathrm{~b}$ and Supplementary Fig. 1). The formation of PPP was confirmed by the Fourier transform infrared (FTIR) spectrometer, which is shown in Supplementary Fig. S2. The red shift of the absorption peak for the stretching vibration of the $\mathrm{C}=\mathrm{O}$ from carboxyl group $\left(1635 \mathrm{~cm}^{-1}\right)$ to amide bond $\left(1689 \mathrm{~cm}^{-1}\right)$ proves the amination of folic acid (FA) molecule (Supplementary Fig. 2a: i and ii). The existence of vibration absorption peaks $\left(3410 \mathrm{~cm}^{-1}\right.$ and $\left.1480 \mathrm{~cm}^{-1}\right)$ for $\mathrm{N}-\mathrm{H}$ bond (Supplementary Fig. 2a: iv) proved the successful synthesis of PLGA-FA. The formation of amide in PPP was also proved in Supplementary Fig. 2b. Soon afterwards $\mathrm{Fe} / \mathrm{FeO}$ NCs were tethered with PPP to form Fe/FeO-PPP heterostructures. Then, DOX and ICG were co-loaded into Fe/FeO-PPP heterostructures to develop flexible DOX-ICG@Fe/FeO-PPP nanocapsules by W/O/W emulsion method, which were used for subsequent experiments ${ }^{35}$. Water-oil ratio $\left(V_{\text {dichloromethane }} / V_{\text {water }}\right)$ for the initial emulsion and PVA are two key conditions in the synthesis of nanocapsules. Water-oil ratio for the initial emulsion could affect the size and hollowness of the nanocapsule (Supplementary Fig. 3a-e), and $V_{\text {dichloromethane }} / V_{\text {water }}=1: 4$ was selected in the following experiments. The presence of PVA could affect the dispersion of nanocapsules (Supplementary Fig. 3f). With the increase of the amount of $\mathrm{Fe} / \mathrm{FeO} \mathrm{NCs}$, the number of NCs on the shell also increased (Supplementary Fig. 4). Finally, we chose to add $3 \mathrm{mg}$ of $\mathrm{Fe} / \mathrm{FeO}$ NCs (Supplementary Fig. 4b).

The core-shell structure of monodispersed Fe/FeO NCs is clearly revealed in transmission electron microscope (TEM) images (Fig. 2a). The Fe/FeO NCs conisted of a core of Fe NPs (of $\sim 8 \mathrm{~nm}$ diameter) with a shell of $\sim 5 \mathrm{~nm}$ thick $\mathrm{FeO}$ as indicated by high-resolution TEM (HRTEM) in Fig. 2b. TEM images of DOX-ICG@PPP and DOX-ICG@Fe/FeO-PPP nanocapsules are presented in Fig. 2c, d, respectively. The average sizes of DOX-ICG@PPP nanocapsules are 203.8 \pm 45.7 nm (Supplementary Fig. 5a) while the DOX-ICG@Fe/FeO-PPP nanocapsules are $218.9 \pm 52.1 \mathrm{~nm}$ (Supplementary Fig. 5b). Furthermore, clear lattice fringes of $0.183 \mathrm{~nm}$ (core) and $0.249 \mathrm{~nm}$ (shell) can be ascribed to the (200) plane of $\mathrm{Fe}$ and (111) plane of $\mathrm{FeO}$, respectively, which are consistent with the X-ray diffraction (XRD) results (Fig. 2e). Element mapping analysis further 
a
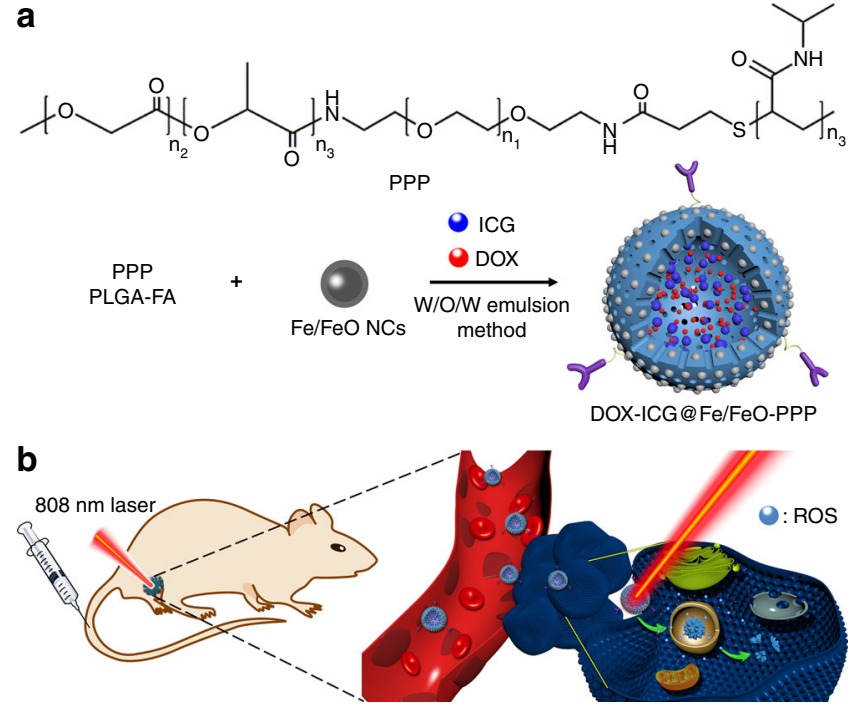

Fig. 1 Synthesis and biomedical application of DOX-ICG@Fe/FeO-PPP nanocapsules. a Fabrication of DOX-ICG@Fe/FeO-PPP nanocapsules. b Schematic representation of nanocapsules for imaging-guided stimuliresponsive chemo/photo- and chemodynamic therapy. PPP Poly (lactic-coglycolic) acid-polyethylene glycol-poly ( $\mathrm{N}$-isopropyl acrylamide)

confirmed the core-shell structure of monodispersed Fe/FeO NCs (Fig. 2b). The presence of satellite peaks in the high-resolution Xray photoelectron spectroscopy (XPS) of Fe $2 \mathrm{p}$ shown in Fig. $2 \mathrm{f}$ demonstrated the existence of $\mathrm{Fe}^{2+}$, supporting the existence of $\mathrm{FeO}$ phase in the $\mathrm{NCs}^{36,37}$. In addition, XPS of $\mathrm{Fe} / \mathrm{FeO} \mathrm{NCs}$ and DOX-ICG@Fe/FeO-PPP nanocapsules are provided in Supplementary Fig. 6 and Supplementary Fig. 7, which could be determined no significant change in the $\mathrm{Fe} / \mathrm{FeO} \mathrm{NCs}$ after compounding with PPP. The values of saturated magnetic intensity for Fe/FeO NCs and DOX-ICG@Fe/FeO-PPP nanocapsules were $74.4 \mathrm{emu} \mathrm{g}^{-1}$ and $14.9 \mathrm{emu} \mathrm{g}^{-1}$ in Supplementary Fig. 8a. Therefore, DOX-ICG@Fe/FeO-PPP nanocapsules are highly expected to be $\mathrm{T}_{2}$-weighted $\mathrm{MRI}$ contrast agents. In addition, the absorption peaks in the NIR region are shown in the UV-vis absorption spectra of the DOX-ICG@Fe/FeO-PPP nanocapsules (Supplementary Fig. 8b), which means the nanocapsules can be the PTT agents (Fig. 1b).

Evaluation and mechanism of the ROS generation. The tumor could be finally destroyed by induced death, which is associated with increased levels of intracellular $\operatorname{ROS}^{38-43} \cdot{ }^{1} \mathrm{O}_{2}$ and $\bullet \mathrm{OH}$ are two important components in ROS. Therefore, the ability of ROS generation of biomedical materials can be estimated through the quantitative detection of the concentrations of $\bullet \mathrm{OH}$ and ${ }^{1} \mathrm{O}_{2}$. The ${ }^{1} \mathrm{O}_{2}$ generation amount of ICG@Fe/FeO-PPP nanocapsules in vitro was evaluated by 1,3-diphenylisobenzofuran (DPBF) with $\mathrm{pH}$ values of 7.4 (Supplementary Fig. 9a), 6.5 (Supplementary Fig. 9b) and 5.4 (Supplementary Fig. $9 \mathrm{c}$ ) ${ }^{44} .{ }^{1} \mathrm{O}_{2}$-generation capability of the nanocapsules at a $\mathrm{pH}$ value of 5.4 was higher than that at 6.5 and 7.4 , because the calculated rate constants were $1.29 \times 10^{-3} \mathrm{~s}^{-1}(\mathrm{pH}=5.4), 3.65 \times 10^{-4}(\mathrm{pH}=6.5)$ and $4.34 \times$ $10^{-5} \mathrm{~s}^{-1}(\mathrm{pH}=7.4)$ (Fig. 3a). Iron-based nanomaterials are reported to start the following reactions (Eqs. 1-3) in $\mathrm{TME}^{40-42}$ :

$$
\begin{gathered}
\mathrm{Fe}^{2+}+\mathrm{H}_{2} \mathrm{O}_{2} \rightarrow \mathrm{Fe}^{3+}+\bullet \mathrm{OH}+\mathrm{OH}^{-} \\
\mathrm{Fe}+2 \mathrm{Fe}^{3+} \rightarrow 3 \mathrm{Fe}^{2+} \\
\mathrm{Fe}^{3+}+\mathrm{H}_{2} \mathrm{O}_{2} \rightarrow \mathrm{Fe}^{2+}+\bullet \mathrm{OOH}+\mathrm{H}^{+}
\end{gathered}
$$

In order to quantitatively evaluate the $\bullet \mathrm{OH}$ production level of ICG@Fe/FeO-PPP nanocapsules, terephthalic acid (TA) oxidation was selected to measure it by fluorescence spectrum (Supplementary Fig. 10 and Supplementary Fig. 11) ${ }^{45}$. It was divided into four groups: (1) TA + Laser; (2) ICG@PPP + TA + Laser; (3) $\mathrm{Fe} / \mathrm{FeO}+\mathrm{TA}+$ Laser; (4) ICG@Fe/FeO-PPP + TA + Laser. These results showed that ICG@Fe/FeO-PPP + TA + Laser group produced maximum fluorescence enhancement at the same time, which confirmed ICG@Fe/FeO-PPP exhibited the strongest ability to produce $\cdot \mathrm{OH}$ in these four groups (Fig. $3 \mathrm{~b}$ ). In addition, the standard consumption of $\mathrm{H}_{2} \mathrm{O}_{2}$ was used to measure the amount of $\bullet \mathrm{OH}$ produced by a spectrophotometric method using copper(II) ion and 2,9-dimethyl-1,10-phenanthroline (DMP) (Supplementary Fig. 12) ${ }^{46}$. UV-vis absorption results in Supplementary Fig. 13 demonstrated the synergistic effect of $\bullet \mathrm{OH}$ production in ICG@Fe/FeO-PPP nanocapsules, comparing with ICG@PPP nanocapsules and Fe/FeO NCs under the irradiation of $808 \mathrm{~nm}$ laser. Electron spin resonance (ESR) spectroscopy further confirmed the potential of the ICG@Fe/FeO-PPP nanocapsules to act as a trigger for the generation of $\bullet \mathrm{OH}$ with 5,5 -dimethyl-1pyrroline $N$-oxide (DMPO) as a spin trap (Fig. $3 \mathrm{c}$ ). The increased ESR signal intensity at lower $\mathrm{pH}$ values indicates the generation of a great number of $\bullet \mathrm{OH}$, because the $\mathrm{Fe} / \mathrm{FeO}$ shell of the ICG@Fe/ FeO-PPP nanocapsules could act as efficient Fenton catalyst in acidic environment ${ }^{47}$. When incubating free ICG and ICG@Fe/ FeO-PPP-FA nanocapsules with oral squamous carcinoma KB cell under the normoxic condition, a similar amount of ROS was generated $1 \mathrm{~min}$ after the irradiation of $808 \mathrm{~nm}$ laser. More interestingly, the ROS level of cells in the hypoxic condition treated with ICG@Fe/FeO-PPP-FA nanocapsules changed only a little in comparison with those under normoxic condition, while cells treated with free ICG showed much lower ROS levels than those under normoxic condition (Fig. 3d). Furthermore, ${ }^{1} \mathrm{O}_{2}$ generation was also evaluated by flow cytometry (Supplementary Figs. 14-16). These results of flow cytometry were consistent with those of confocal fluorescence imaging result in Fig. 3d. Additionally, we proposed an explanation of the ROS production of ICG@Fe/FeO-PPP-FA nanocapsules in tumor cells from two aspects (Fig. 3e). On the one hand, Fe/FeO NCs on the surface of the nanocapsules produced large amounts of $\bullet \mathrm{OH}$ by catalyzation of $\mathrm{H}_{2} \mathrm{O}_{2}$ in TME. On the other hand, the ICG inside the nanocapsules generated a certain amount of ${ }^{1} \mathrm{O}_{2}$ by PDT process under the irradiation of $808 \mathrm{~nm}$ laser. The two synergistic factors ensure that the nanocapsules could produce ROS even in the hypoxia area of tumor through interaction.

DOX delivery evaluation of DOX-ICG@Fe/FeO-PPP nanocapsules. DOX was loaded into the nanocapsules by $\mathrm{W} / \mathrm{O} / \mathrm{W}$ emulsion method. The amount of loaded DOX was quantified by UV-vis absorption spectrum, which could show a distinguished absorption peak appearing at the wavelength of $481 \mathrm{~nm}$ (Supplementary Fig. 17). The saturated loading capacity of DOX-ICG@Fe/FeO-PPP nanocapsules is $18.3 \%$ by calculation (Supplementary Fig. 18), illustrating that ICG@Fe/FeO-PPP nanocapsules are good candidates as drug carriers.

The photothermal efficiency of ICG@Fe/FeO-PPP nanocapsules was investigated under $808 \mathrm{~nm}$ laser irradiation based on the UV-vis absorbance spectra of the nanocapsules (Supplementary Fig. 19a). For an optimal concentration (of just $60 \mathrm{mg} \mathrm{L}^{-1}$ ) of nanocapcules, the laser power of $0.3 \mathrm{~W} \mathrm{~cm}^{-2}$ was employed to obtain a most suitable treatment temperature of $51.5^{\circ} \mathrm{C}$ (Supplementary Fig. 19b and c). Interestingly, the ICG@Fe/ FeO-PPP nanocapsules were able to maintain good photothermal effects even after several irradiation cycles (Supplementary Fig. 19d). During the laser irradiation process, the diameters of 
a

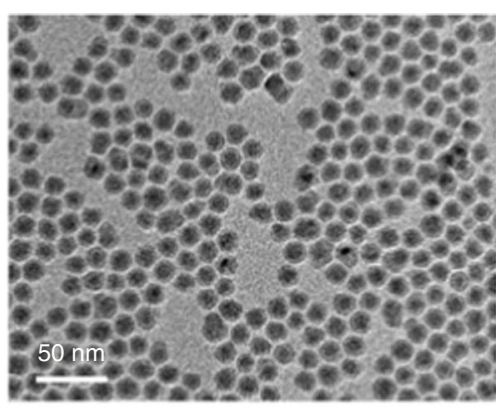

C

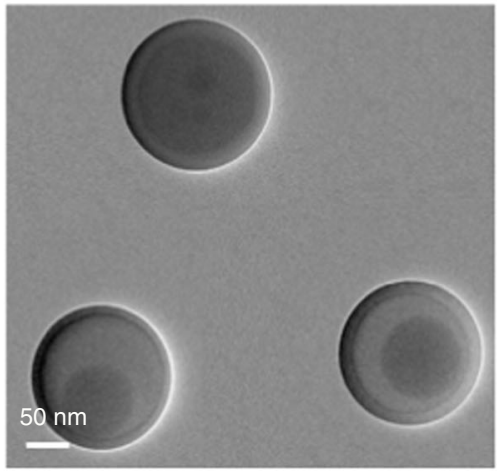

e

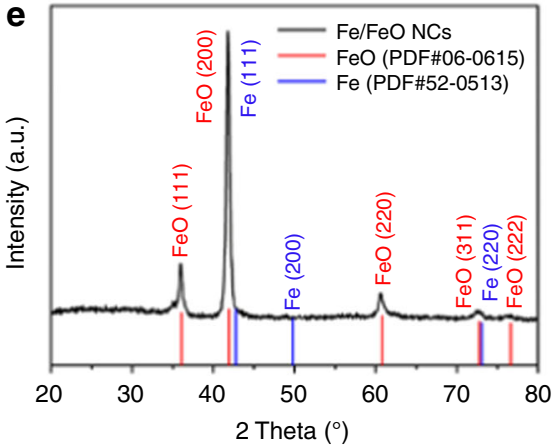

b

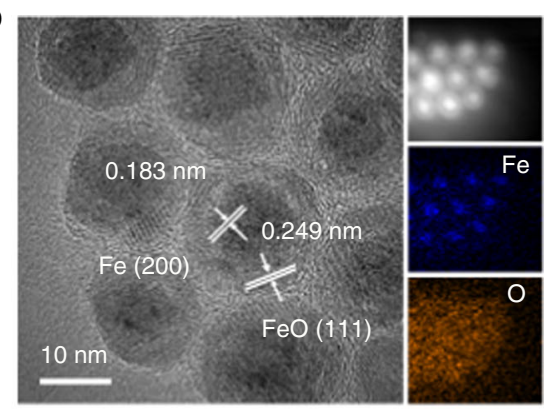

d
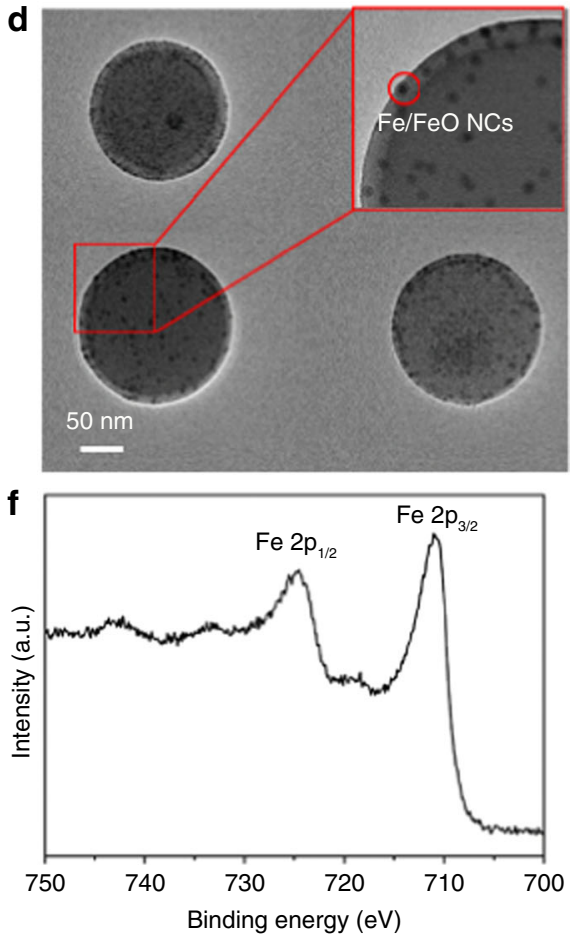

Fig. 2 Characterization of DOX-ICG@Fe/FeO-PPP nanocapsules. a TEM image of Fe/FeO NCs. b HRTEM image of Fe/FeO NCs, inset: EDS-mapping of $\mathrm{Fe} / \mathrm{FeO} \mathrm{NCs}$. c TEM image of DOX-ICG@PPP nanocapsules, d TEM image of DOX--ICG@Fe/FeO-PPP nanocapsules. Inset: HRTEM image of DOX-ICG@Fe/FeO-PPP nanocapsules. e XRD patterns of Fe/FeO NCs. f High-resolution XPS of Fe 2p for Fe/FeO NCs

nanocapsules were contracted sustainably, which enabled the nanocapsules to release DOX in large amounts due to high temperature stimuli (Supplementary Figs. 19e, f and 20). These results proved that the nanocapsules successfully produced a significant temperature increase under laser irradiation.

To evaluate the release efficiency of DOX-ICG@Fe/FeO-PPP nanocapsules, these nanocapsules were incubated in a phosphate buffer with $\mathrm{pH} 7.4,6.5$ or 5.4 under the $808 \mathrm{~nm}$ laser irradiation. In Fig. 4a, DOX-ICG@PPP nanocapsules did not show obvious difference in drug release at $\mathrm{pH} 7.4,6.5$ or 5.4. However, DOX-ICG@Fe/FeO-PPP nanocapsules released up to $79.0 \%$ at $\mathrm{pH} 5.4$ after 80 -h treatment, while the release was limited to $67.4 \%$ at $\mathrm{pH} 6.5$ and $55.4 \%$ at $\mathrm{pH} 7.4$. The increase of drug release of DOX-ICG@Fe/FeO-PPP nanocapsules is associated to the instability of $\mathrm{Fe} / \mathrm{FeO} \mathrm{NCs}$ in weak acidic condition. Meanwhile, DOX-ICG@Fe/FeO-PPP nanocapsules and DOX-PPP@PPP nanocapsules were exposed to laser irradiation for $5 \mathrm{~min}$ (laser on) in a buffer with $\mathrm{pH} 7.4,6.5$ or 5.4 and then incubated for 55 min (Fig. 4b). DOX-ICG@PPP nanocapsules did not respond to the laser irradiation and $\mathrm{pH}$ stimuli, which corresponded with the above results $(\mathrm{pH}=7.4$ : 20.4\%, $\mathrm{pH}=6.5: 23.4 \%, \mathrm{pH}=5.4: 26.0 \%$ ), while abrupt release from DOX-ICG@Fe/FeO-PPP nanocapsules was detected at
pH 7.4 (42.2\%), $6.5(58.2 \%)$ and 5.4 (73.9\%) when NIR irradiation was repeated after every $1 \mathrm{~h}$.

Dual-stimulus responsive ICG@Fe/FeO-PPP nanocapsules. The TEM images of ICG@Fe/FeO-PPP nanocapsules show obvious shrinkage from $220.9 \pm 25.5 \mathrm{~nm}$ to $161.9 \pm 21.8 \mathrm{~nm}$ at $\mathrm{pH}$ 6.5 in $24 \mathrm{~h}$ upon laser irradiation, which demonstrates the effect of irradiation on the morphology of nanocapsules (Fig. 4c and Supplementary Fig. 21). After $48 \mathrm{~h}$, small size nanocapsules $(54.5 \pm 4.1 \mathrm{~nm})$ were observed as presented in the TEM images in Fig. 4c. Based on the above results, we deduced that the radial stress analysis of flexible DOX-ICG@Fe/FeO-PPP nanocapsules causes the shrinking process (Fig. 4d). Subsequently, the change of ICG@Fe/FeO-PPP nanocapsules within 1 week after laser irradiation were measured by TEM at pH 6.5 (Supplementary Fig. 22). Moreover, bio-TEM images of KB cells incubated with ICG@Fe/FeO-PPP nanocapsules was also provided in Supplementary Fig. 23. These results proved the instability of ICG@Fe/ FeO-PPP nanocapsules after laser irradiation in weak acidic condition. We hypothesized that the stability of this system was caused by large-scale local buckling instability of the nanocapsules after the irradiation of the laser ${ }^{48}$. The irradiation of the 
a

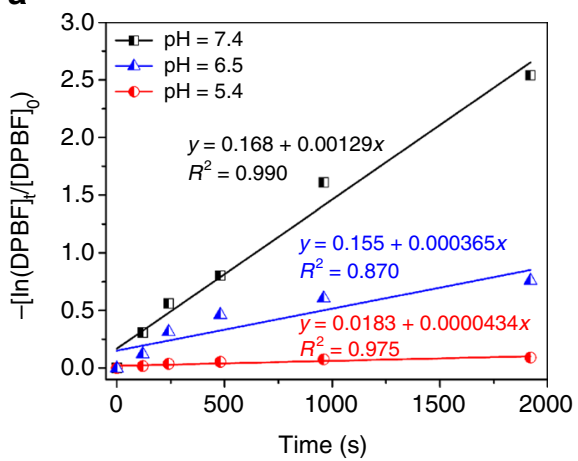

d

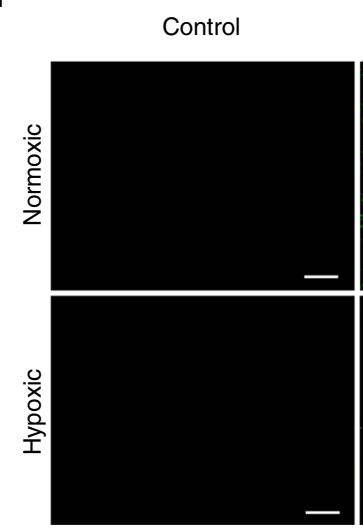

b

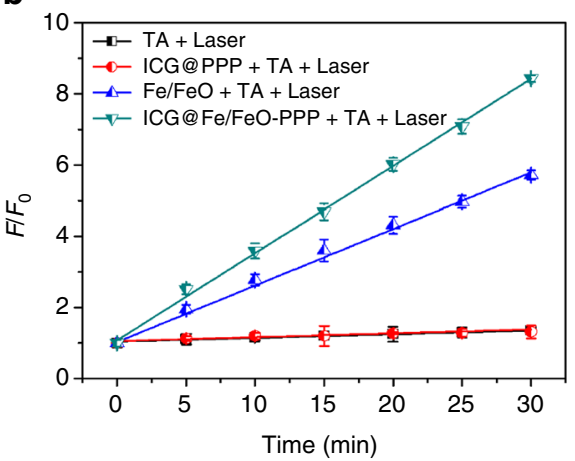

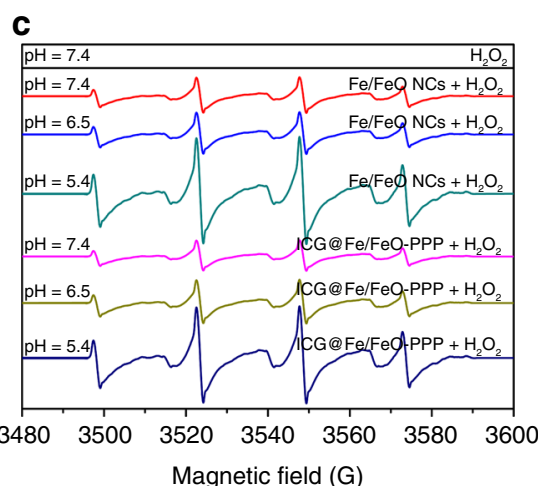

e
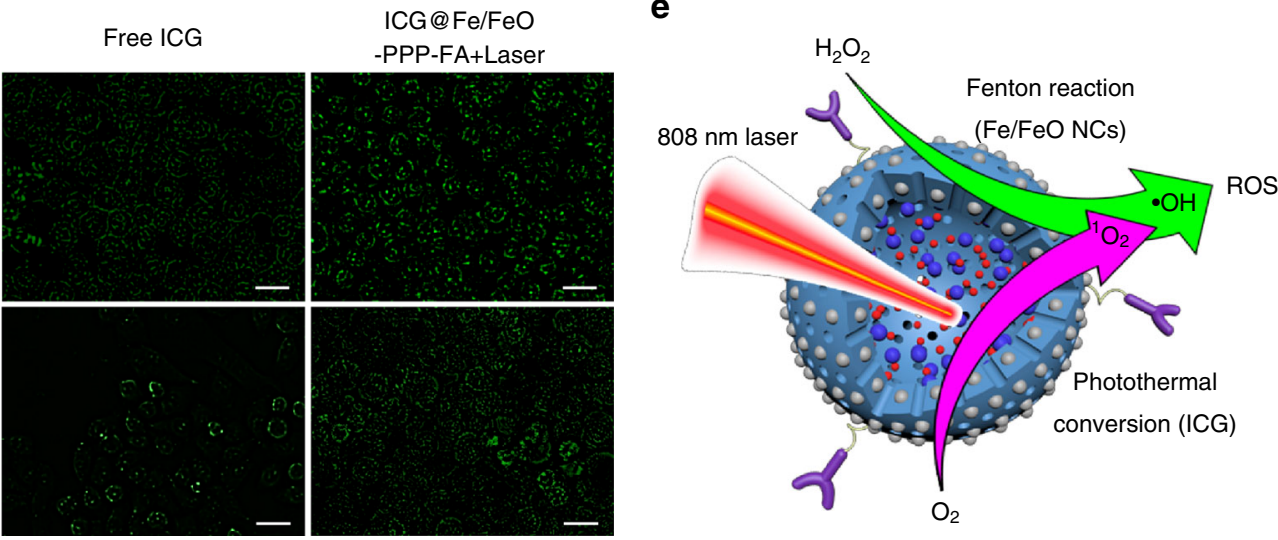

Fig. 3 Evaluation of the ROS generation and the mechanism of ROS production. a Detection of ${ }^{1} \mathrm{O}_{2}$ by UV-vis absorbance spectra for ICG@Fe/FeO-PPP nanocapsules with DPBF at pH value of 7.4, 6.5 and 5.4 phosphate buffer. b Fluorescence intensity of TAOH at $440 \mathrm{~nm}$ as a function of laser irradiation time for the ICG@Fe/FeO-PPP nanocapsules and different control treatments. $F_{0}$ and $F$ were the fluorescence intensities of the system without or with treatment, respectively. The error bars represent the standard deviations of three separate measurements. c ESR spectra of Fe/FeO NCs and ICG@Fe/ FeO-PPP nanocapsules with DMPO as the spin trap in different $\mathrm{pH}$ values condition $\left(7.4,6.5\right.$ and 5.4 ). d ${ }^{1} \mathrm{O}_{2}$ generation evaluated by $\mathrm{DHR} 123$ in $\mathrm{KB}$ cells treated with free ICG and ICG@Fe/FeO-PPP-FA nanocapsules under normoxic and hypoxic conditions (scale bars: $20 \mu \mathrm{m}$ ). e Synergism schematic of

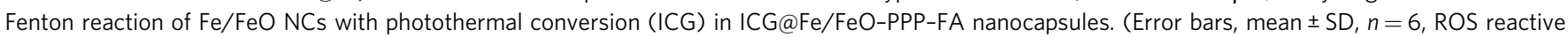
oxygen species.)

laser could lead to the temperature increase inside of the nanocapsule, which further caused the change in interaction between the internal interface of nanocapsule and water molecule by intermolecular forces of $\mathrm{PPP}^{49,50}$. Due to the degradability of ester bond and amide bond in PPP at pH below 6.5 (Fig. 4e), the shrinking nanocapsules were eventually hydrolyzed ${ }^{51-56}$.

In vivo imaging and biodistribution. The cytotoxicity and photothermal ability of ICG@Fe/FeO-PPP nanocapsules at cellular level were evaluated by using NIH3T3 and KB cells. Due to the overexpression of the folate receptor in KB cells, ICG@Fe/ FeO-PPP-FA nanocapsules can specifically recognize the $\mathrm{KB}$ cells through interactions between FA and folate receptor (on the surface of cells). Supplementary Fig. 24a shows that the cell viability of NIH3T3 and KB cells, incubated with the ICG@Fe/FeOPPP-FA nanocapsules (of $\mathrm{Fe}$ concentrations up to $3.20 \mathrm{mM}$ ), could reach even higher than $95 \%$, which is clear indication of very low cytotoxicity of nanocapsules. Subsequently, we examined the photothermal efficiency of each nanocapsules in $\mathrm{KB}$ cells. As a result, a more significant laser-induced photothermal killing efficiency of KB cells was observed in ICG@Fe/FeO-PPP-FA nanocapsules with the irradiation of laser (Supplementary Fig. 24b). Furthermore, live/dead cell staining experiments were used to evaluate the laser-triggered phototherapy effect. As shown in Supplementary Fig. 24c, red fluorescence appeared only for the cells treated with DOX-ICG@Fe/FeO-PPP-FA nanocapsules under the irradiation with laser, which is consistent with the CCK8 assay results in Supplementary Fig. 24b. These results demonstrated that DOX-ICG@Fe/FeO-PPP-FA nanocapsules can specifically kill the KB cells by PTT.

Iron NPs have the potential to be the agents for $\mathrm{T}_{2}$-weighted MRI. The $r_{2}$ value of ICG@Fe/FeO-PPP nanocapsules was around $130.7 \mathrm{mM}^{-1} \mathrm{~s}^{-1}$ when dispersed in water and decreased to $75.9 \mathrm{mM}^{-1} \mathrm{~s}^{-1}$ when incubated with $\mathrm{KB}$ cells (Supplementary Fig. 25). We also assessed the $\mathrm{T}_{2}$-weighted MRI capability in vivo after intravenous injection of ICG@Fe/FeO-PPP and ICG@Fe/ FeO-PPP-FA nanocapsules $\left(20 \mathrm{mg} \mathrm{kg}^{-1}, 200 \mathrm{~mL}\right)$ into $\mathrm{KB}$ tumor-bearing nude mice. Figure $5 \mathrm{a}, \mathrm{b}$ clearly indicates that the ICG@Fe/FeO-PPP-FA nanocapsules show more stronger signal intensity and make the tumor darker than ICG@Fe/FeO-PPP after $24 \mathrm{~h}$ of injection. These results suggest higher accumulations of ICG@Fe/FeO-PPP-FA at the tumor sites owing to the active targeting.

Furthermore, fluorescence imaging was carried out to track the in vivo behaviors of ICG@Fe/FeO-PPP and ICG@Fe/ FeO-PPP-FA nanocapsules $\left(20 \mathrm{mg} \mathrm{kg}^{-1}, 200 \mathrm{~mL}\right)$ after intravenous injection into tumor model. ICG could act as fluorescent molecule with the excitation wavelength of $780 \mathrm{~nm}$ and emission wavelength of $810 \mathrm{~nm}$ (Supplementary Fig. 26). Strong intensity of fluorescence signals were mainly showed for ICG@Fe/ FeO-PPP-FA nanocapsules in the tumor site after $12 \mathrm{~h}$ (Fig. 5c). In contrast, no obvious fluorescence signal appeared in the tumor site for ICG@Fe/FeO-PPP nanocapsules even after $24 \mathrm{~h}$. 
a

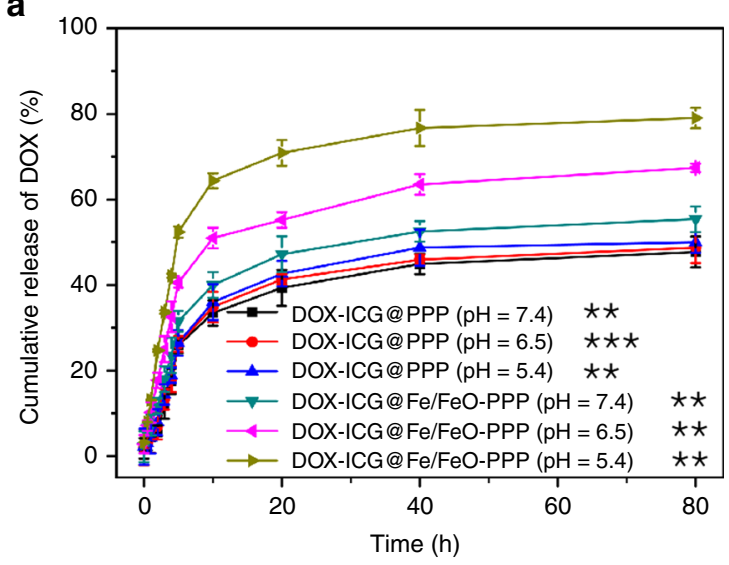

c
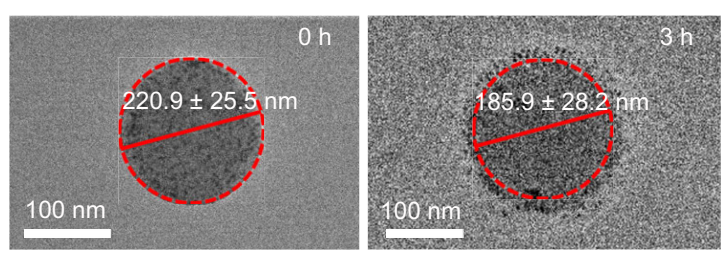

b
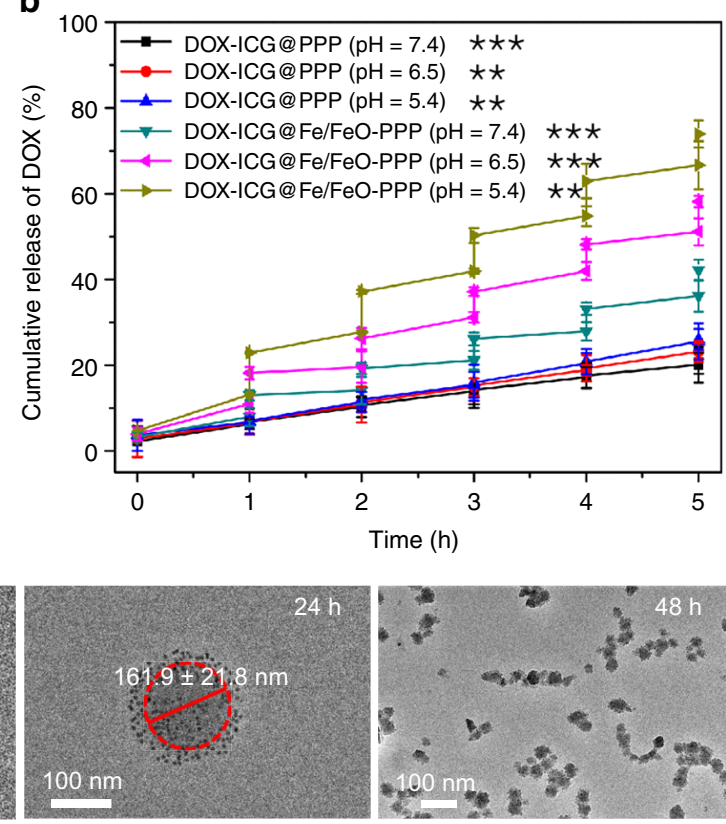

d

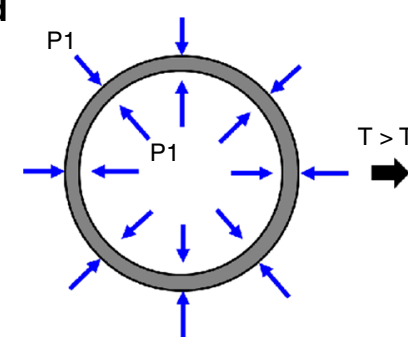

Structure stabele state

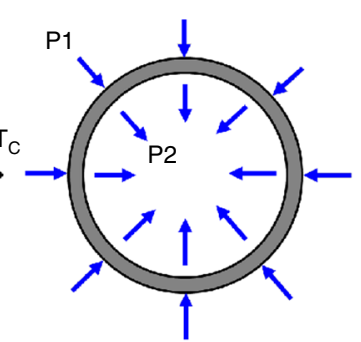

Structure unstabele state

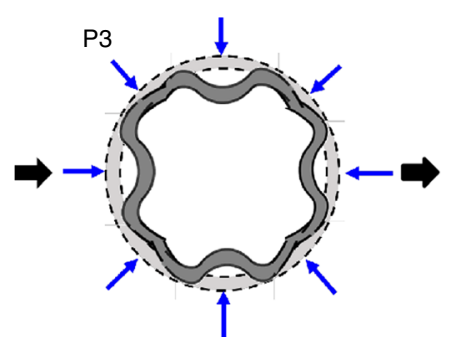

Large-scale local buckling instability .

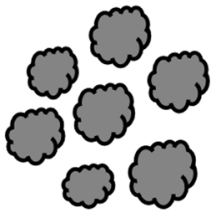

Hydrolytic state

e

(1)

(2)

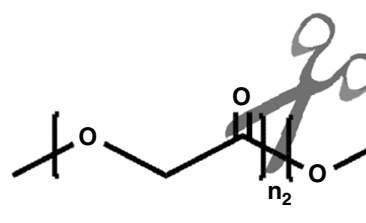

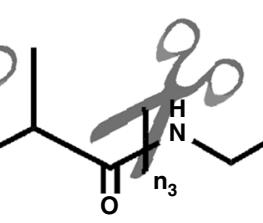

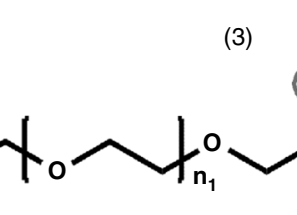

(3)

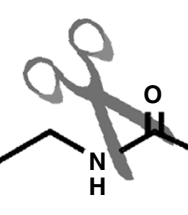

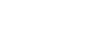


a

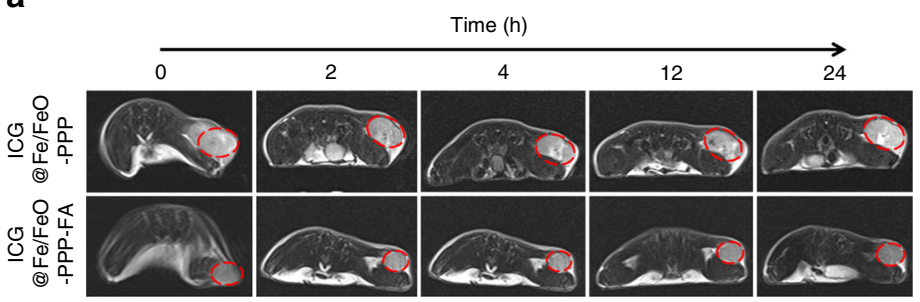

C

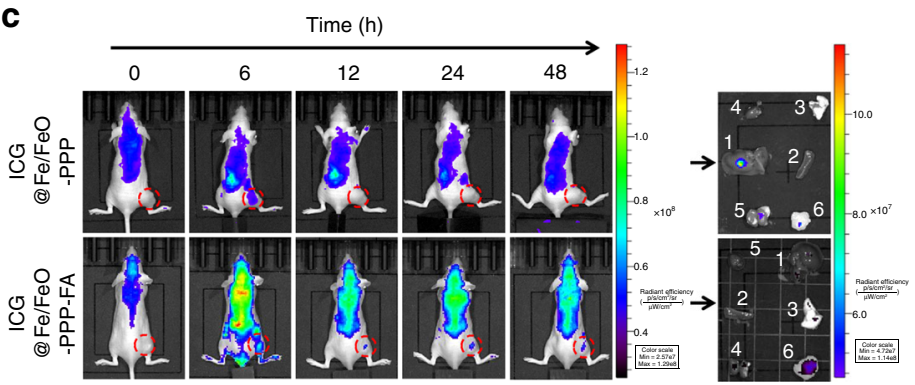

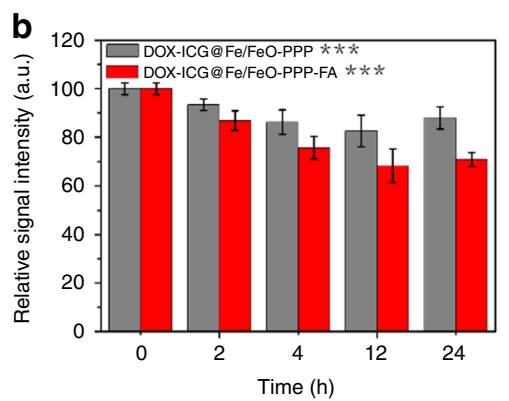

d

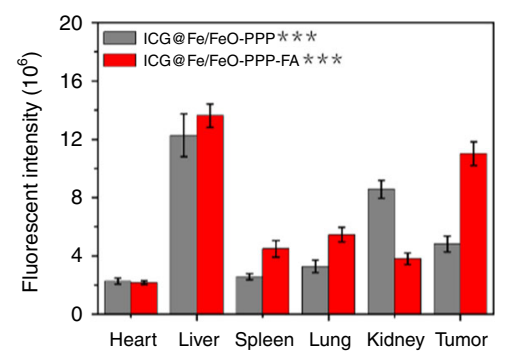

Fig. 5 In vivo imaging. a Real-time MRI of KB tumor-bearing mice after intravenous injection of ICG@Fe/FeO-PPP nanocapsules and ICG@Fe/FeO-PPP-FA nanocapsules. b The relative MRI signal intensities changing at the tumor site after intravenous injection of ICG@Fe/FeO-PPP nanocapsules and ICG@Fe/ FeO-PPP-FA nanocapsules, respectively. c Real-time fluorescence images of tumor-bearing mice after intravenous injection of ICG@Fe/FeO-PPP nanocapsules and ICG@Fe/FeO-PPP-FA nanocapsules. Ex vivo fluorescence images of liver (1), spleen (2), lung (3), heart (4), kidney (5) and tumor (6), which were obtained at $48 \mathrm{~h}$ post-injection. $\mathbf{d}$ The fluorescence intensities of the major organs after intravenous injection of ICG@Fe/FeO-PPP

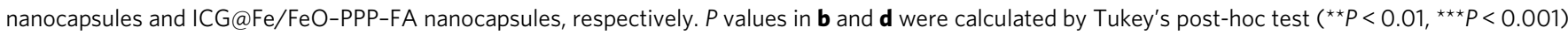
by comparing ICG@Fe/FeO-PPP nanocapsules with ICG@Fe/FeO-PPP-FA nanocapsules. Error bars, mean \pm SD $(n=5)$

mice, the local temperature of the tumor site rapidly increases from $37^{\circ} \mathrm{C}$ to $50.4^{\circ} \mathrm{C}$ within $5 \mathrm{~min}$, but for the mice treated with DOX-ICG@Fe/FeO-PPP nanocapsules, the temperature only reaches to $45.2^{\circ} \mathrm{C}$ (Fig. 6b and Supplementary Fig. 28). These results again confirmed the superior targeting capability of DOX-ICG@Fe/FeO-PPP-FA nanocapsules as proved in the bioimaging process. Furthermore, the bio-distribution of nanocapsules after intravenous injection for 3 days was detected by ICP-MS and confirmed the targeting capacity of DOX-ICG@Fe/ FeO-PPP-FA nanocapsules in vivo (Supplementary Fig. 29). Comparing with the other six groups (ICG@Fe/FeO-PPP-FA nanocapsules and laser irradiation, ICG@Fe/FeO-PPP nanocapsules and laser irradiation, only DOX-ICG@Fe/FeO-PPP-FA nanocapsules, only ICG@Fe/FeO-PPP-FA nanocapsules, saline and laser irradiation, and only saline), the excellent antitumor efficiency of DOX-ICG@Fe/FeO-PPP-FA nanocapsules was demonstrated by tumor volume with significant inhibition and elimination in vivo (Fig. 6c). The growth status of representative nude mice in each group at the time interval of $0,3,6,9,12,15$, 18 days throughout the treatment cycle was observed (Fig. 6e). The tumor of harvested mice injected with only DOX-ICG@Fe/ FeO-PPP-FA nanocapsules under the laser irradiation $(808 \mathrm{~nm}$, $0.3 \mathrm{~W} \mathrm{~cm}^{-2}$ ) was completely eradicated after treatment. An obvious damage was evidenced to the tumor cells of mice by cell necrosis and apoptosis in the group of injection with DOX-ICG@Fe/FeO-PPP-FA nanocapsules after laser irradiation. Mice treated with other groups showed less necrotic areas (Fig. 6f and Supplementary Fig. 30). These results showed that DOX-ICG@Fe/FeO-PPP-FA nanocapsules were efficient as targeting nanomaterials with antitumor capacity in KB-bearing mice models.

Subsequently, toxicity analysis of these nanocapsules was investigated. There was no decrease in the weight of the mice in each group during the treatment which demonstrates the low toxicity of the ICG@Fe/FeO-PPP-FA nanocapsules (Fig. 6d). As displayed in Supplementary Fig. S31, no significant difference in the levels of these liver and kidney function indicators, including alanine aminotransferase (ALT), aspartate aminotransferase (AST), alkaline phosphatase (ALP), creatinine (CRE) and blood urea nitrogen (BUN), between the treatment and control groups was observed. These results indicate the good hepatic and kidney safety profile of each group. Finally, the histological analysis was done by hematoxylin and eosin (H\&E) staining of the main organs after the treatment to study the damage in acute and chronic stages. No tissue necrosis was observed in the main organs (heart, liver, spleen, lung and kidney) for the seven groups, demonstrating that the formulations mentioned above have no obvious biological toxicity (Supplementary Fig. S32).

\section{Discussion}

In summary, we have constructed an intelligent NIR/TME dualresponsive nanocapsule (made of DOX-ICG@Fe/FeO-PPP) for enhanced tumor accumulation and improved therapy efficacy. The large initial size of these nanocapsules ensures the circulatory stability in the blood while, under irradiation of an NIR laser, the shrinkage and decomposition of nanocapsule in acidic TME guarantees intratumoral permeability of NPs and the controllable release of DOX. Interestingly, the overproduced ROS by synergistic catalysis of Fenton reaction based on Fe/FeO NCs and light activation from ICG relieves the hypoxia for solid tumors, which is necessarily required to mitigate the hypoxia-related resistance during chemo/photo- and chemodynamic therapy. As a result of these unique properties of nanocapsules, almost complete destruction of tumors was realized. In addition, dual-mode MRI and fluorescence imaging provide complementary imaging information. Hence, this study presents the design of smart nanocapsules with enhanced tumor accumulation, highly effective therapy and diagnosis to accelerate exploitation and clinical translation of intelligent theranostics nanocapsules. 
$808 \mathrm{~nm}$ laser $\quad 808 \mathrm{~nm}$ laser

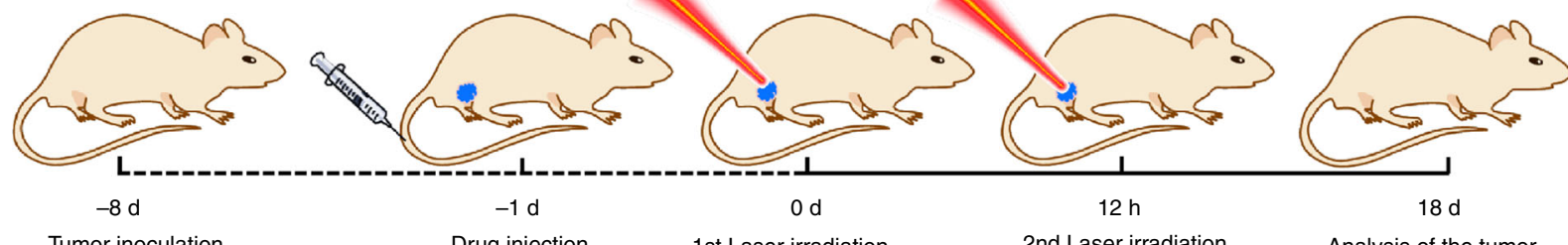

Tumor inoculation

Drug injection

1st Laser irradiation

2nd Laser irradiation

Analysis of the tumor

b
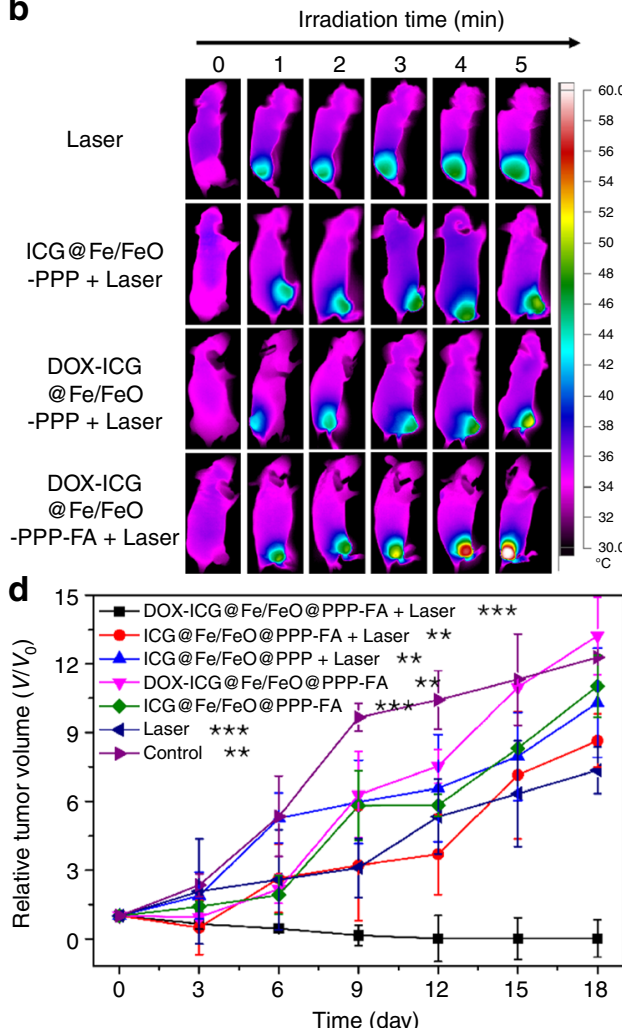

e

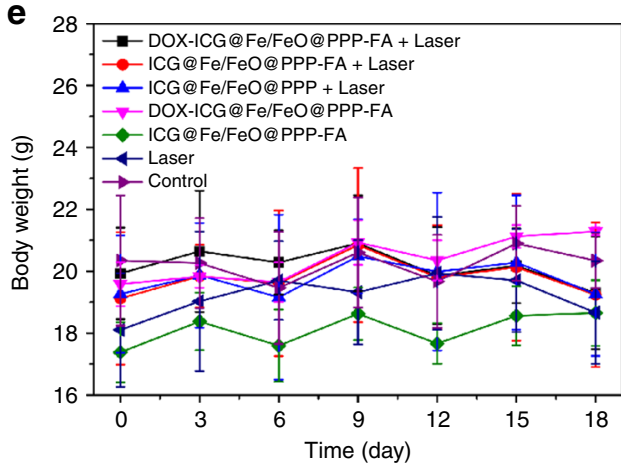

Control

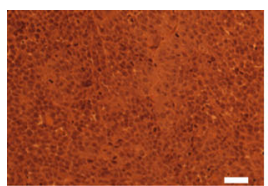

Laser

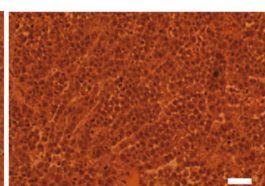

C
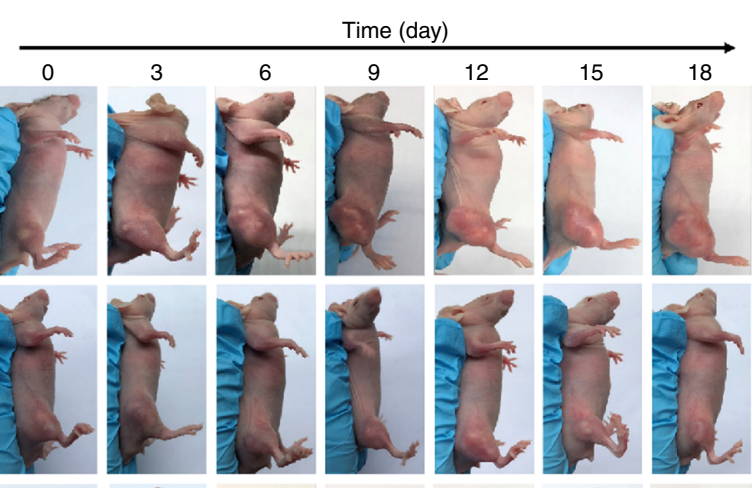

ICG@

Fe/FeO-PPP-FA
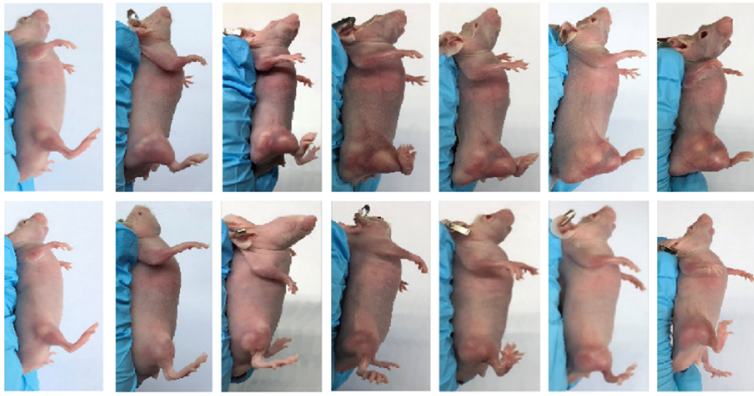

DOX-ICG@ $\mathrm{Fe} / \mathrm{FeO}-\mathrm{PPP}+\mathrm{FA}$
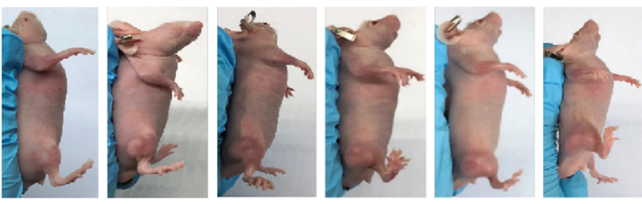

ICG@

$\mathrm{Fe} / \mathrm{FeO}-\mathrm{PPP}$

+ Laser
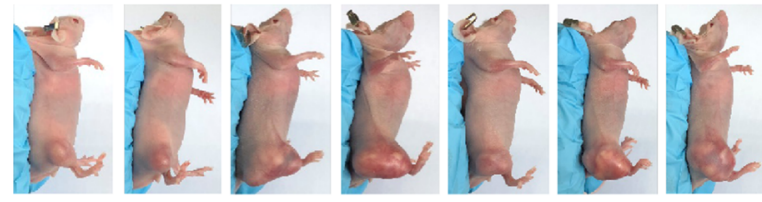

ICG@ $\mathrm{Fe} / \mathrm{FeO}-\mathrm{PPP}-\mathrm{FA}$ + Laser
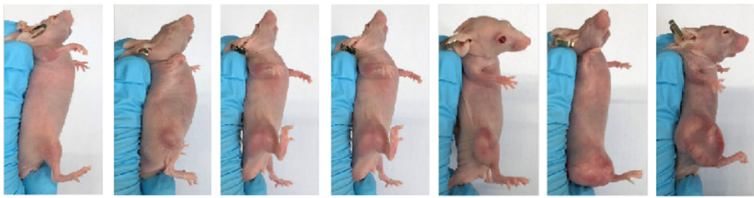

DOX-ICG@ Fe/FeO-PPP-FA

+ Laser
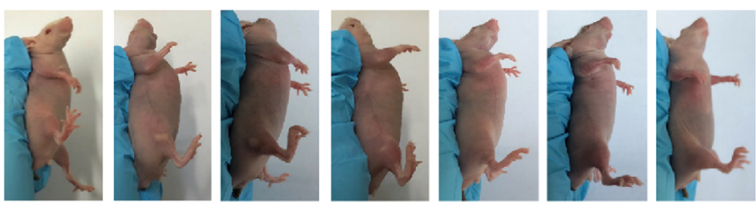

ICG@ $\mathrm{Fe} / \mathrm{FeO}-\mathrm{PPP}$

DOX-ICG@
Fe/FeO-PPP-FA

+ Laser
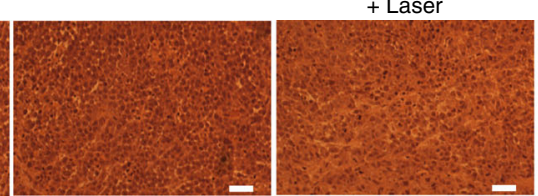

ICG@

DOX-ICG@

$\mathrm{Fe} / \mathrm{FeO}-\mathrm{PPP}-\mathrm{FA}$

+ Laser

\section{Methods}

Materials. Copper (II) chloride dehydrate $\left(\mathrm{CuCl}_{2} \cdot 2 \mathrm{H}_{2} \mathrm{O}, 99.9 \%\right)$, potassium bromide $\left(\mathrm{NH}_{4} \mathrm{Br}, 99 \%\right)$ and TA $(99.9 \%)$ were purchased from J\&K Chemicals. Oleylamine (OAm, tech. $70 \%$ ), oleic acid (OA, 99\%), poly( $N$-isopropylacrylamide) (PNIPAM, carboxylic acid terminated, $\mathrm{Mr}=5000$ ), dicyclohexylcarbodiimide (DCC), $N$-hydroxysuccinimide (NHS), FA, DPBF, DMP, DMPO, calcein-AM and propidium iodide (PI) were purchased from Sigma-Aldrich. Iron pentacarbonyl was from Tianyi Co. Ltd, Jiangsu, China. Dihydrorhodamine 123 (DHR123) was purchased from Thermo Fisher Scientific (Waltham, MA, USA). $\mathrm{H}_{2} \mathrm{O}_{2}$ was obtained from Beijing Chemicals Inc. (Beijing, China). Octadecene (ODE, tech. 90\%) was purchased from Alfa Asear. All the chemicals were used without additional purification, except dimethyl sulfoxide (DMSO), $\mathrm{CHCl}_{3}$, triethylamine, and 
Fig. 6 Therapeutic effect of DOX-ICG@Fe/FeO-PPP-FA nanocapsules. a Schematic illustration of DOX-ICG@Fe/FeO-PPP-FA nanocapsules-based tumor therapy. b Real-time thermal IR images of KB tumor-bearing mice after intravenous injection of DOX-ICG@Fe/FeO-PPP nanocapsules and DOX-ICG@Fe/ FeO-PPP-FA nanocapsules under $808 \mathrm{~nm}$ laser irradiation $\left(0.3 \mathrm{~W} \mathrm{~cm}^{-2}, 5 \mathrm{~min}\right)$. c Volume change of tumor in the different treatment (five mice per group). $\mathbf{d}$ Body weight change of mice in the different treatment (five mice per group). e Representative photograph of excised tumors from euthanatized mice at 18 days post treatment (five mice per group). $\mathbf{f} \mathrm{H} \& \mathrm{E}$-stained images of tumor regions with different treatments after 2 days of intravenous injection (scale bars: $50 \mu \mathrm{m}) . P$ values in c were calculated by Tukey's post-hoc test $\left({ }^{\star \star} P<0.01,{ }^{\star \star \star} P<0.001\right)$ by comparing other groups with the last group (DOX-ICG@Fe/FeO-PPP-FA + Laser). Error bars, mean \pm SD $(n=5)$

were performed under argon utilizing a homemade heating apparatus, four-necked bottles (Synthware), a glove box (MIKROUNA) and $\mathrm{Ar} / \mathrm{N}_{2} /$ vacuum lines. $\mathrm{H}_{2} \mathrm{~N}$-PEG- $\mathrm{NH}_{2}(\mathrm{Mr}=4000)$, PEG-3,4-dihydroxy benzyl amine (DIB-PEG- $\left.\mathrm{NH}_{2}\right)$ were synthesized according to oxyamination and aldimine condensation ${ }^{60-62}$. All the dialysis bags $(\mathrm{Mr}=8000-14,000)$ were obtained from Shanghai Med.

Instruments. Varian $400 \mathrm{MHz}$ NMR was used to acquire ${ }^{1} \mathrm{H}-\mathrm{NMR}$ spectra. The conventional bright-field images were obtained utilizing FEI Tecnai T20 microscope $(200 \mathrm{kV})$, and HRTEM was carried out on an FEI Tecnai F30 microscope $(300 \mathrm{kV})$. Reinforced carbon membrane support grid was used to obtain the EDS mapping. XPS measurements were performed on an imaging X-ray photoelectron spectrometer using $\mathrm{Al} \mathrm{Ka}$ radiation (Axis Ultra DLD, Kratos Analytical Ltd.). All the collected spectra were calibrated with contaminated C 1s peak at $284.8 \mathrm{eV}$, and were analyzed using CasaXPS software (2.3.12 Dev7). PANalytical X'Pert3 powder $\mathrm{X}$-ray diffractometer was used to obtain X-ray diffraction (PXRD) patterns with $\mathrm{Cu}-\mathrm{Ka}(\lambda=0.15405 \mathrm{~nm})$ radiation at $40 \mathrm{kV}$ and $40 \mathrm{~mA}$. Nicolet FTIR spectrometer (Magna-IR 750) was used for FTIR measurements. Physical property measurement system (PPMS-9, Quantum Design, USA) was used to measure the magnetization. EPR spectra were measured by Bruker A300-9.5/12 spectrometer at room temperature. Fluorescent microscope (Leica DR) equipped with a digital camera (ORCA-ER, Hamamatsu) was used to obtain fluorescence microscopic images. Inductively coupled plasma-atomic emission spectrometer (ICP-AES, Prodigy 7, and Leeman, USA) was used to quantify the concentrations of Fe. Dynamic light scattering (DLS) was measured using a particle size analyzer (Zetasizer Nano ZS90, Malvern, England). UV 1750 spectrophotometer (Shimadzu, Japan) was used to measure the UV-vis absorbance. Infrared thermal imaging instrument (FLIR A325SC camera) was used to record the temperature detection and thermal image; $808 \mathrm{~nm}$ high-power multimode pump laser (Shanghai Connect iber Optics Co.) was used for NIR laser.

Synthesis of PLGA-FA. PLGA-FA was synthesized by the amidation between PLGA and ethylenediamine-derivatized FA (FA- $\left.\mathrm{NH}_{2}\right)$. Typically, PLGA (15000.0 $\mathrm{mg}, 0.1 \mathrm{mmol}$ ) and FA- $\mathrm{NH}_{2}(48.5 \mathrm{mg}, 0.1 \mathrm{mmol})$ were dissolved in $50 \mathrm{~mL}$ of dry DMSO. Subsequently, DCC $(0.492 \mathrm{~g}, 2.4 \mathrm{mmol})$ and NHS $(23.0 \mathrm{mg}, 0.2 \mathrm{mmol})$ were added. The reaction mixture was stirred for $14 \mathrm{~h}$ at room temperature in the dark. Finally, the reaction product was isolated and purified by recrystallization. The yield of FA- $\mathrm{NH}_{2}: 60 \%$. MS: $m / z=482[\mathrm{M}+\mathrm{H}]^{+}$. $1 \mathrm{H}$ NMR (DMSO-d6, 300 $\mathrm{MHz}, \delta$ in ppm): $186-1.99$ (m, 2H, C21-H), 2.01-2.06 (m, 2H, C22-H), 2.21-2.38 (m, 2H, C25-H), 2.45 (m, 2H, C26-H), $4.34(\mathrm{dd}, J=5.4,9.36 \mathrm{~Hz}, 1 \mathrm{H}, \mathrm{C} 19-\mathrm{H}), 4.49$ (s, $2 \mathrm{H}, \mathrm{C} 9-\mathrm{H} 2), 6.66(\mathrm{~d}, J=8.7 \mathrm{~Hz}, 2 \mathrm{H}), 7.66(\mathrm{~d}, J=8.7 \mathrm{~Hz}, 2 \mathrm{H}), 8.65(\mathrm{~s}, 1 \mathrm{H})$

Synthesis of PLGA-PEG-NH 2 . PLGA-PEG- $\mathrm{NH}_{2}$ was synthesized by the amidation between PLGA and $\mathrm{H}_{2} \mathrm{~N}-\mathrm{PEG}-\mathrm{NH}_{2} \cdot \mathrm{H}_{2} \mathrm{~N}-\mathrm{PEG}-\mathrm{NH}_{2}$ was synthesized by oxyamination method ${ }^{61,62}$. Subsequently, PLGA $(15000.0 \mathrm{mg}, 0.1 \mathrm{mmol})$ and $\mathrm{H}_{2} \mathrm{~N}-\mathrm{PEG}-\mathrm{NH}_{2}(400.0 \mathrm{mg}, 0.1 \mathrm{mmol})$ were dissolved in $50 \mathrm{~mL}$ of dry DMSO. Subsequently, DCC $(24.6 \mathrm{mg}, 0.12 \mathrm{mmol})$ and NHS $(23.0 \mathrm{mg}, 0.2 \mathrm{mmol})$ were added. The reaction mixture was stirred for $14 \mathrm{~h}$ at room temperature in the dark. Finally, the reaction product was isolated and purified by recrystallization.

\section{Synthesis of PPP. PPP was synthesized by the amidation between}

PLGA-PEG-NH ${ }_{2}$ and PNIPAM. Briefly, PLGA-PEG-NH $\mathrm{NH}_{2}(1900.0 \mathrm{mg}, 0.1 \mathrm{mmol})$ and PNIPAM $(500.0 \mathrm{mg}, 0.1 \mathrm{mmol})$ were dissolved in $50 \mathrm{~mL}$ of dry DMSO. Subsequently, DCC (24.6 mg, $0.12 \mathrm{mmol}$ ) and NHS $(23.0 \mathrm{mg}, 0.2 \mathrm{mmol})$ were added to the system. The reaction mixture was stirred for $14 \mathrm{~h}$ at room temperature in the dark. Finally, the reaction product was isolated and purified by recrystallization.

Synthesis of Fe/FeO NCs. Fe/FeO NCs were synthesized by a facile seedmediated growth method. Firstly, $12 \mathrm{~nm}$ Fe NCs were synthesized following our previously reported method ${ }^{63}$. In the typical synthesis, ODE $(62.5 \mathrm{mmol}), \mathrm{NH}_{4} \mathrm{Br}$ $(0.1 \mathrm{mmol})$ and $\mathrm{OAm}(1 \mathrm{mmol})$ were mixed under a gentle $\mathrm{N}_{2}$ flow for $1 \mathrm{~h}$ in a four-necked flask. Then the solution was heated to $100^{\circ} \mathrm{C}$ and kept for $1 \mathrm{~h}$ to remove the organic impurities. $\mathrm{Fe}(\mathrm{CO})_{5}(5 \mathrm{mmol})$ was injected into the reaction system when the temperature reached $180^{\circ} \mathrm{C}$ and kept for $30 \mathrm{~min}$. After the system cooled down to room temperature, $27 \mathrm{ml}$ of acetone was added to the system. After centrifugation, the product was washed by ethanol and hexane. The obtained $\mathrm{Fe}$ NCs were dispersed in $2 \mathrm{~mL}$ of dichloromethane. Next, $\mathrm{Fe}(\mathrm{acac})_{3}(0.706 \mathrm{~g}, 4 \mathrm{mmol})$,
OA ( $4 \mathrm{~mL}, 12.5 \mathrm{mmol}), \mathrm{OAm}(6 \mathrm{~mL}, 17.5 \mathrm{mmol})$ and the resulted bcc-Fe NPs were mixed in a four-necked flask. Then the solution was heated to $120^{\circ} \mathrm{C}$ and kept for $1 \mathrm{~h}$ to remove the organic impurities. The solution was then heated to $220^{\circ} \mathrm{C}$ and kept for $30 \mathrm{~min}$. After $30 \mathrm{~min}$, the system was heated to $300^{\circ} \mathrm{C}$ and kept for $10 \mathrm{~min}$ under a $\mathrm{N}_{2}$ blanket. The solution was cooled down to room temperature and the NPs were washed by ethanol and hexane. Finally, the $\mathrm{Fe} / \mathrm{FeO} \mathrm{NCs}$ were dispersed in hexane.

Synthesis of nanocapsules. DOX-ICG@Fe/FeO-PPP nanocapsules were formulated using W/O/W emulsion method. Typically, DOX (10.0 mg) and ICG (3 $\mathrm{mg}$ ) were dissolved in $5 \% \mathrm{w} / \mathrm{v}$ PVA solution $(2 \mathrm{~mL}$ ) by using ultrasound for $10 \mathrm{~min}$. $\mathrm{Fe} / \mathrm{FeO} \mathrm{NCs}(3 \mathrm{mg})$ and PPP $(25.0 \mathrm{mg})$ were dissolved in $8 \mathrm{~mL}$ of dichloromethane for $10 \mathrm{~min}$. Then, the two were mixed and kept for $10 \mathrm{~min}$ by using ultrasound. The $\mathrm{O} / \mathrm{W}$ emulsion was then added to $5 \% \mathrm{w} / \mathrm{v}$ PVA solution $(40 \mathrm{~mL})$ to evaporate the organic solvent at room temperature for $4 \mathrm{~h}$. DOX-ICG@Fe/FeO-PPP nanocapsules were obtained after centrifugation at $6300 \times \mathrm{g}$ for $10 \mathrm{~min}$. These synthesized DOX-ICG@Fe/FeO-PPP nanocapsules were treated with lyophilization for further use. DOX-ICG@Fe/FeO-PPP-FA nanocapsules, ICG@Fe/FeO-PPP nanocapsules, ICG@Fe/FeO-PPP-FA nanocapsules and ICG@PPP nanocapsules were synthesized by using the same method as DOX-ICG@Fe/FeO-PPP nanocapsules, The only difference was that the ratio of PLGA-FA:PPP was 5:95 in the synthesis of DOX-ICG@Fe/FeO-PPP-FA nanocapsules and ICG@Fe/FeO-PPP-FA nanocapsules.

Photothermal effect of ICG@Fe/FeO-PPP nanocapsules. A total of $350 \mu \mathrm{L}$ of ICG@Fe/FeO-PPP nanocapsule dispersions with different concentrations $(0,10$, $20,40,60$ and $\left.80 \mathrm{mg} \mathrm{L}^{-1}\right)$ were irradiated with a laser $\left(808 \mathrm{~nm}, 0.3 \mathrm{~W} \mathrm{~cm}^{-2}\right)$ for $5 \mathrm{~min}$, and their temperature in solution was recorded by an online type thermocouple thermometer. Similarly, in order to study the influence of optical density on photothermal conversion, $350 \mu \mathrm{L}$ of $60 \mathrm{mg} \mathrm{L}^{-1}$ ICG@Fe/FeO-PPP nanocapsule dispersions were irradiated with an 808-nm laser with different power densities $\left(0.2,0.4,0.6,0.8\right.$ and $\left.1.0 \mathrm{~W} \mathrm{~cm}^{-2}\right)$ for $5 \mathrm{~min}$. The change of temperature in solution was recorded by an online type thermocouple thermometer. The photostability of ICG@Fe/FeO-PPP nanocapsule dispersions $\left(60 \mathrm{mg} \mathrm{L}^{-1}\right)$ was estimated by irradiating in a quartz cuvette with a laser $\left(808 \mathrm{~nm}, 0.3 \mathrm{~W} \mathrm{~cm}^{-2}\right)$ for $5 \mathrm{~min}$ and then cooling to room temperature without irradiation. The photostability was tested by repeating such processes four times.

Cell culture. NIH3T3 and KB cell lines were obtained from the Cancer Institute and Hospital of the Chinese Academy of Medical Science. All cell-culture-related reagents were purchased from Invitrogen. RPMI-1640 culture medium supplemented with $10 \% \mathrm{FBS}$ and $1 \%$ penicillin/streptomycin was used to culture cells at $37^{\circ} \mathrm{C}$ under $5 \% \mathrm{CO}_{2}$ with $100 \%$ humidity.

In vitro cytotoxicity assay. $\mathrm{NIH} 3 \mathrm{~T} 3$ or $\mathrm{KB}$ cells $(1 \times 104$ cells per well $)$ seeded into a 96-well cell culture plate were incubated with ICG@Fe/FeO-PPP nanocapsules in different Fe concentrations $(0,0.01,0.10,0.20,0.40,0.80,1.60$ and $3.20 \mathrm{mM})$ for $48 \mathrm{~h}$ at $37^{\circ} \mathrm{C}$ under $5 \% \mathrm{CO}_{2}$. The relative cell viabilities were determined by a standard CCK- 8 viability assay $(n=3)$.

In vitro photothermal ablation of KB cells. NIH3T3 and $\mathrm{KB}$ cells $\left(1 \times 10^{4}\right.$ cells per well) seeded into a 96-well cell culture plate were incubated with ICG@Fe/ FeO-PPP-FA nanocapsules, DOX-ICG@Fe/FeO-PPP-FA nanocapsules, ICG@Fe/FeO-PPP nanocapsules and laser irradiation, ICG@Fe/FeO-PPP-FA nanocapsules and laser irradiation, DOX-ICG@Fe/FeO-PPP-FA nanocapsules and laser irradiation in Fe concentrations of $0,0.01,0.10,0.20,0.40,0.80,1.60$ and $3.20 \mathrm{mM}$ for $24 \mathrm{~h}$ at $37^{\circ} \mathrm{C}$ under $5 \% \mathrm{CO}_{2}$, respectively. The cells were washed three times with PBS and fed with fresh medium, followed by irradiating with laser for $5 \mathrm{~min}\left(808 \mathrm{~nm}, 0.3 \mathrm{~W} \mathrm{~cm}^{-2}\right)$, respectively. Finally, the viability of cells was evaluated by a standard CCK- 8 assay $(n=3)$.

To examine the photothermal effect of ICG@Fe/FeO-PPP-FA nanocapsules on $\mathrm{KB}$ cells in vitro, $\mathrm{KB}$ cells seeded $\left(1 \times 10^{4}\right.$ cells per well $)$ in culture dishes were incubated with ICG@Fe/FeO-PPP-FA nanocapsules and laser irradiation, ICG@Fe/FeO-PPP nanocapsules and laser irradiation, DOX-ICG@Fe/ FeO-PPP-FA nanocapsules only, ICG@Fe/FeO-PPP-FA nanocapsules only, laser irradiation only and control (without any treatment) for $4 \mathrm{~h}$, respectively. Laser 
( $808 \mathrm{~nm}, 0.3 \mathrm{~W} \mathrm{~cm}^{-2}$ ) was used to irradiate the adherent cell solution. After DMEM was removed, the cells were washed with PBS three times. Calcein-AM $(100 \mu \mathrm{L})$ and PI solution $(100 \mu \mathrm{L})$ were incubated with $\mathrm{KB}$ cells for $15 \mathrm{~min}$. Living cells were stained with calcein-AM (green fluorescence) and dead cells with PI (red fluorescence) solution $(n=3)$.

In vitro •OH generation of ICG@Fe/FeO-PPP nanocapsules. The $\cdot \mathrm{OH}$ gener ated by ICG@Fe/FeO-PPP nanocapsules was detected by TA oxidation method. The whole process was based on the fluorescence spectrum because after the oxidation of TA to TAOH by $\bullet \mathrm{OH}$, nonfluorescent TA was converted to fluorescent $\mathrm{TAOH}$. The experiment was divided into four groups: (1) only under $808 \mathrm{~nm}$ laser irradiation $\left(0.3 \mathrm{~W} \mathrm{~cm}^{-2}\right)$, (2) ICG@PPP nanocapsules under $808 \mathrm{~nm}$ laser irradiation $\left(0.3 \mathrm{~W} \mathrm{~cm}^{-2}\right),(3) \mathrm{Fe} / \mathrm{FeO}$ under $808 \mathrm{~nm}$ laser irradiation $\left(0.3 \mathrm{~W} \mathrm{~cm}^{-2}\right)$ and $(4)$ ICG@Fe/FeO-PPP nanocapsules under $808 \mathrm{~nm}$ laser irradiation $\left(0.3 \mathrm{~W} \mathrm{~cm}{ }^{-2}\right)$. Firstly, TA solution was prepared $(1 \mathrm{mM})$; subsequently, different groups were treated accordingly, and the fluorescence spectra at different times $(0,5,10,15,20$, 25 and $30 \mathrm{~min}$ ) were measured $(\mathrm{ex} / \mathrm{em}=327 \mathrm{~nm} / 437 \mathrm{~nm})$. Laser irradiation time was $5 \min (n=6)$.

In vitro ${ }^{1} \mathbf{O}_{\mathbf{2}}$ generation of nanocapsules. The ${ }^{1} \mathrm{O}_{2}$ generation potential of all nanomaterials in vitro was detected by using DPBF. Briefly, $0.5 \mathrm{~mL}$ of the sample suspension $\left(C_{\mathrm{Fe}}=0.10 \mathrm{mM}, C_{\text {nanocapsules }}=60 \mathrm{mg} \mathrm{L}^{-1}\right)$ was added to $0.5 \mathrm{~mL}$ of DPBF $(0.02 \mathrm{mM})$ solution (phosphate buffer, $\mathrm{pH}$ 5.4). The mixture was irradiated by laser $\left(808 \mathrm{~nm}, 0.3 \mathrm{~W} \mathrm{~cm}^{-2}\right)$ for $5 \mathrm{~min}(n=6)$. The concentration of DPBF was determined by measuring the absorbance at $466 \mathrm{~nm}$ as a function of time using a $\mathrm{UV}$-vis absorption spectra. The rate constant for ${ }^{1} \mathrm{O}_{2}$ generation by ICG@PPP nanocapsules, $\mathrm{Fe} / \mathrm{FeO} \mathrm{NCs}$ and ICG@Fe/FeO-PPP nanocapsules was calculated using the following Eq. (4):

$$
\operatorname{In}\left(\frac{C_{t}}{C_{0}}\right)=\operatorname{In}\left(\frac{A_{t}}{A_{0}}\right)=-k t
$$

where $C_{t}$ is the concentration of DPBF at a certain time point; $C_{0}$ is the concentration of DPBF at the initial time point; $A_{t}$ is the absorbance at the wavelength of $466 \mathrm{~nm}$ at a certain time point; $A_{0}$ is the absorbance at the wavelength of $466 \mathrm{~nm}$ at the initial time point; $k$ is the reaction rate constant, which was calculated by correlated fitting linear equation;and $t$ is the mixing time for DPBF and each nanomaterial.

Evaluation of ROS generation in KB cells. ROS-generating capabilities of free ICG and ICG@Fe/FeO-PPP-FA nanocapsules under normoxic or hypoxic condition were assessed by DHR123, respectively ${ }^{11}$. DHR123 staining was carried out as follows: cells were incubated with ICG $(0.05 \mathrm{mM})$ or ICG@Fe/FeO-PPP-FA nanocapsules $\left(60 \mathrm{mg} \mathrm{L}^{-1}\right)$ for $24 \mathrm{~h}$. Then, $1 \mu \mathrm{g}$ of DHR123 was added to cell media under laser irradiation $\left(808 \mathrm{~nm}, 0.3 \mathrm{~W} \mathrm{~cm}^{-2}\right)$ for $1 \mathrm{~min}$. Confocal laser scanning microscopy (CLSM) and flow cytometry were used to observe the fluorescence intensity by DHR $123(\mathrm{ex} / \mathrm{em}=488 \mathrm{~nm} / 520 \mathrm{~nm}, n=3)$.

DOX delivery evaluation of DOX-ICG@Fe/FeO-PPP nanocapsules. $5 \mathrm{mg}$ of DOX-ICG@Fe/FeO-PPP nanocapsules DOX loading and releasing of DOX-ICG@Fe/FeO-PPP nanocapsules. A total of $5 \mathrm{mg}$ of DOX-ICG@Fe/ FeO-PPP nanocapsules were added in $10 \mathrm{~mL}$ of dichloromethane under ultrasound for $1 \mathrm{~h}$. The absorbance at the wavelength of $481 \mathrm{~nm}$ for the DOX from the decomposed DOX-ICG@Fe/FeO-PPP nanocapsules was measured by UV-vis absorption spectra $(n=6)$. Loading of DOX in DOX-ICG@Fe/FeO-PPP nanocapsules was quantified using the simulation of standard working curve for the detection of DOX. Loading capacity was calculated using the following Eq. (5):

$$
\text { Encapsulation efficiency }(\%)=\frac{M_{\text {DOX loaded }}}{M_{\text {nanocapsules }}} \times 100 \% \quad 5
$$

where $M_{\mathrm{DOX}}$ loaded is the mass of DOX detected after the treatment of dichloromethane and $M_{\text {nanocapsules }}$ is the total mass of nanocapsules.

The release of DOX from DOX-ICG@Fe/FeO-PPP nanocapsules was evaluated by dialyzing the nanocapsules in the dark in phosphate buffer at $\mathrm{pH} 7.4,6.5$ and 5.4 for different times at $37^{\circ} \mathrm{C}$ in $80 \mathrm{~h}$. UV-vis absorption spectra were used to determine the released DOX at the wavelength of $481 \mathrm{~nm}$. For the $808 \mathrm{~nm}$ lasertriggered DOX release, DOX-ICG@Fe/FeO-PPP nanocapsules were dialyzed in the buffer solution with $\mathrm{pH}$ values of $7.4,6.5$ or 5.4 at $37^{\circ} \mathrm{C}$. Released DOX was collected at the time points of $1,2,3,4$ and $5 \mathrm{~h}$. At the time periods of $1,2,3$, and $4 \mathrm{~h}$, a laser $\left(808 \mathrm{~nm}, 0.3 \mathrm{~W} \mathrm{~cm}^{-2}\right)$ was employed for $5 \mathrm{~min}$. The released DOX was collected immediately and the dosage was measured using simulation of standard working curve for the detection of DOX. In addition, the release of DOX from DOX-ICG@PPP nanocapsules was followed as the same method above $(n=6)$.

Animals and tumor model. All experiments involving animals were performed in accordance with the guidelines of the Institutional Animal Care and Use Committee (IACUC) of Peking University, Beijing, China. Four- to five-week-old Balb/c nude mice with the average weight of $20 \mathrm{~g}$ were provided by the Beijing Center for Disease Control and Prevention, Beijing, China. SPF animal house was provided to mice under a $12-\mathrm{h}$ light and $12-\mathrm{h}$ darkness cycle and were fed a standard laboratory diet and tap water ad libitum. KB cells $(0.2 \mathrm{~mL}$ cells in 1640 culture medium without FBS) were injected into the mice subcutaneously at the right axillary region.

In vivo MRI. For $\mathrm{MRI}$ in vivo, the $\mathrm{KB}$-tumor-bearing mice were intravenously injected with ICG@Fe/FeO-PPP nanocapsules and ICG@Fe/FeO-PPP-FA nanocapsules $\left(20 \mathrm{mg} \mathrm{kg}^{-1}, 200 \mu \mathrm{L}\right)$. After the injection, $\mathrm{T}_{2}$ images were obtained at 0,6 12,24 and $48 \mathrm{~h}$ by a clinic 3T MRI scanner (Philips, TR $=1200 \mathrm{~ms}, \mathrm{TE}=30.2 \mathrm{~ms}$ slice thickness $=2.5 \mathrm{~mm}$ ). The intensity of the MRI signal before injection was used as the control $(n=5)$

In vivo fluorescence imaging. The KB-tumor-bearing mice were intravenously injected with ICG@Fe/FeO-PPP nanocapsules and ICG@Fe/FeO-PPP-FA nanocapsules $\left(20 \mathrm{mg} \mathrm{kg}^{-1}, 200 \mu \mathrm{L}\right)$ for fluorescence imaging in vivo. The fluorescence signal was recorded by the CRi maestro ex in vivo imaging system (USA) at $0,6,12,24$ and $48 \mathrm{~h}$ after the injection. The fluorescence signal before injection was used as the control. To confirm the in vivo distribution of ICG@Fe/ FeO-PPP nanocapsules and ICG@Fe/FeO-PPP-FA nanocapsules, mice were sacrificed $48 \mathrm{~h}$ post-injection. The main organs (liver, heart, lung, spleen, tumor and kidneys) were collected for imaging and semi-quantitative biodistribution analysis $(n=5)$.

In vivo antitumor efficiency evaluation. Mice bearing $200 \mathrm{~mm}^{3} \mathrm{~KB}$ tumors were randomly divided into seven groups: (1) DOX-ICG@Fe/FeO-PPP-FA nanocapsules and laser irradiation; (2) ICG@Fe/FeO-PPP-FA nanocapsules and laser irradiation; (3) ICG@Fe/FeO-PPP nanocapsules and laser irradiation; (4) only DOX-ICG@Fe/FeO-PPP-FA nanocapsules; (5) only ICG@Fe/FeO-PPP-FA nanocapsules; (6) saline and laser irradiation and (7) control (only saline). Five mice were contained in each group. After $200 \mathrm{~mL}$ of saline or nanocapsules $(20 \mathrm{mg}$ $\mathrm{kg}^{-1}$ ) were intravenously injected into nude mice bearing the KB tumor for $24 \mathrm{~h}$, mice were exposed to $808 \mathrm{~nm}$ laser $\left(0.3 \mathrm{~W} \mathrm{~cm}^{-2}\right)$ for $5 \mathrm{~min}$ (Fig. 5a) at the first time. Subsequently, the second irradiation by $808 \mathrm{~nm}$ laser $\left(0.3 \mathrm{~W} \mathrm{~cm}{ }^{-2}, 5 \mathrm{~min}\right)$ was started after $12 \mathrm{~h}$. The changes in body weight and tumor volume during 18 days of treatment period were recorded $(n=5)$

In vivo blood biochemistry test. Mice were randomly divided into five groups: (1) DOX-ICG@Fe/FeO-PPP-FA nanocapsules and laser irradiation; (2) ICG@Fe/ FeO-PPP-FA nanocapsules and laser irradiation; (3) ICG@Fe/FeO-PPP nanocapsules and laser irradiation; (4) only DOX-ICG-Fe/FeO-PPP-FA nanocapsules and (5) control (only saline). After $200 \mathrm{~mL}$ of nanocapsules or saline $\left(20 \mathrm{mg} \mathrm{kg}^{-1}\right)$ were intravenously injected into nude mice for $72 \mathrm{~h}$, the blood biochemistry test was started, which included five important hepatic and kidney function indicators (ALT, AST, ALP, CRE and BUN) $(n=5)$.

Histological evaluation. Mice from each group were euthanized, then major organs and tumor were recovered, followed by fixing with $10 \%$ neutral buffered formalin after 18 days treatment. The organs were embedded in paraffin and sectioned at $5 \mathrm{~mm}$; H\&E or Prussian blue staining was performed for histological examination. The slides were observed under an optical microscope $(n=5)$.

Statistical analysis. Statistical analysis was calculated by Tukey's post-hoc test with statistical significance assigned at ${ }^{* *} P<0.01$ (moderately significant), ${ }^{* * *} P<$ 0.001 (highly significant).

Ethical approval. All experiments involving animals were performed in accordance with the guidelines of the Institutional Animal Care and Use Committee (IACUC) of Peking University, Beijing, China.

Original data are provided in a Source Data file.

Reporting summary. Further information on experimental design is available in the Nature Research Reporting Summary linked to this paper.

\section{Data availability}

The source data underlying Figs. 2e, f, 3a, b, 4a-c, 5b, d, 6c, d and Supplementary Figs. 2a, b, 5a, b, 6a-d, 7a-f, 8a, b, 9a-c, 10a-d, 12a, b, 13a, b, 17a, b, 18, 19a-d, 21a-d, 24a, b, 25, 26, 27b, 28, 29 and 31 are provided as a Source Data file. All the other data supporting the findings of this study are available within the article and its Supplementary Information files and from the corresponding authors upon reasonable request. A reporting summary for this article is available as a Supplementary Information file.

Received: 24 January 2019; Accepted: 19 August 2019; Published online: 27 September 2019 


\section{References}

1. Blanco, E. et al. Principles of nanoparticle design for overcoming biological barriers to drug delivery. Nat. Biotechnol. 33, 941-951 (2015).

2. Couvreur, P. Nanoparticles in drug delivery: past, present and future. $A d v$. Drug Del. Rev. 65, 21-23 (2013).

3. Khan, A. et al. Palladium-catalyzed decarboxylative cycloaddition of vinylethylene carbonates with formaldehyde: enantioselective construction of tertiary vinylglycols. Angew. Chem. Int. Ed. 126, 6557-6560 (2014).

4. Chen, D. et al. Biodegradable, hydrogen peroxide and glutathione dual responsive nanoparticles for potential programmable paclitaxel release. J. Am. Chem. Soc. 140, 7373-7376 (2018).

5. Jain, R. K. et al. Delivering nanomedicine to solid tumors. Nat. Rev. Clin. Oncol. 7, 653-664 (2010).

6. Petros, R. A. et al. Strategies in the design of nanoparticles for therapeutic applications. Nat. Rev. Drug Discov. 9, 615-627 (2010).

7. Sun, Q. et al. Integration of nanoassembly functions for an effective delivery cascade for cancer drugs. Adv. Mater. 26, 7615-7621 (2014).

8. Song, G. et al. Perfluorocarbon-loaded hollow Bi2Se3 nanoparticles for timely supply of oxygen under near-infrared light to enhance the radiotherapy of cancer. Adv. Mater. 28, 2716-2723 (2016).

9. Prasad, $\mathrm{P}$. et al. Multifunctional albumin- $\mathrm{MnO}_{2}$ nanoparticles modulate solid tumor microenvironment by attenuating hypoxia, acidosis, vascular endothelial growth factor and enhance radiation response. ACS Nano $\mathbf{8}$, 3202-3212 (2014).

10. Chen, $\mathrm{H}$. et al. $\mathrm{H}_{2} \mathrm{O}_{2}$-activatable and $\mathrm{O}_{2}$-evolving nanoparticles for highly efficient and selective photodynamic therapy against hypoxic tumor cells. $J$. Am. Chem. Soc. 137, 1539-1547 (2015).

11. Kim, J. et al. Continuous $\mathrm{O}_{2}$-evolving $\mathrm{MnFe}_{2} \mathrm{O}_{4}$ nanoparticle-anchored mesoporous silica nanoparticles for efficient photodynamic therapy in hypoxic cancer. J. Am. Chem. Soc. 139, 10992-10995 (2017).

12. Wu, W. et al. Hemoglobin-based oxygen carriers combined with anticancer drugs may enhance sensitivity of radiotherapy and chemotherapy to solid tumors. Artif. Cells Blood Substit. Biotechnol. 37, 163-165 (2009).

13. Huang, K. et al. Size-dependent Localization and penetration of ultrasmall gold nanoparticles in cancer cells, multicellular spheroids, and tumors in vivo. ACS Nano 6, 4483-4493 (2012).

14. Wang, J. et al. The role of micelle size in tumor accumulation, penetration, and treatment. ACS Nano 9, 7195-7206 (2015).

15. Choi, H. S. et al. Renal clearance of quantum dots. Nat. Biotechnol. 25 1165-1170 (2007).

16. Li, J. et al. Dual endogenous stimuli-responsive polyplex micelles as smart two-step delivery nanocarriers for deep tumor tissue penetration and combating drug resistance of cisplatin. J. Mater. Chem. B 2, 1813-1824 (2014).

17. Chen, Q. et al. Intelligent albumin- $\mathrm{MnO}_{2}$ nanoparticles as $\mathrm{pH}-/ \mathrm{H}_{2} \mathrm{O}_{2}$ responsive dissociable nanocarriers to modulate tumor hypoxia for effective combination therapy. Adv. Mater. 28, 7129-7136 (2016).

18. Bai, J. et al. A facile ion-doping strategy to regulate tumor microenvironments for enhanced multimodal tumor theranostics. J. Am. Chem. Soc. 140, 106-109 (2018).

19. Choi, H. S. et al. Design considerations for tumour-targeted nanoparticles. Nat. Nanotechnol. 5, 42-47 (2009).

20. Semenza, G. L. Targeting HIF-1 for cancer therapy. Nat. Rev. Cancer 3 721-732 (2003).

21. Semenza, G. L. Hypoxia-inducible factors: mediators of cancer progression and targets for cancer therapy. Trends Pharmacol. Sci. 33, 207-214 (2012).

22. Lou, Y. et al. Targeting tumor hypoxia: suppression of breast tumor growth and metastasis by novel carbonic anhydrase IX inhibitors. Cancer Res. 71, 3364-3376 (2011).

23. Rademakers, S. E. et al. Molecular aspects of tumour hypoxia. Mol. Oncol. 2, 41-53 (2008).

24. Cheng, Y. et al. Perfluorocarbon nanoparticles enhance reactive oxygen levels and tumour growth inhibition in photodynamic therapy. Nat. Commun. 6, 8785 (2015).

25. Cheng, Y. et al. Deep penetration of a PDT drug into tumors by noncovalent drug-gold nanoparticle conjugates. J. Am. Chem. Soc. 133, 2583-2591 (2011)

26. Zhang, C. et al. Synthesis of iron nanometallic glasses and their application in cancer therapy by a localized Fenton reaction. Angew. Chem. Int. Ed. 55, 2101-2106 (2016).

27. Zhang, C. et al. Marriage of scintillator and semiconductor for synchronous radiotherapy and deep photodynamic therapy with diminished oxygen dependence. Angew. Chem. Int. Ed. 54, 1770-1774 (2015).

28. Shen, M. et al. Preparation of a thermosensitive gel composed of a mPEG PLGA-PLL-cRGD nanodrug delivery system for pancreatic tumor therapy. ACS Appl. Mater. Interfaces 7, 20530-20537 (2015).

29. $\mathrm{Su}, \mathrm{Z}$. et al. Adverse reaction in patients with drug allergy history after simultaneous intravenous fundus fluorescein angiography and indocyanine green angiography. J. Ocul. Pharmacol. Ther. 28, 410-413 (2012).
30. Knecht, P. B. et al. Indocyanine green angiography-guided management of Vogt-Koyanagi-Harada disease: differentiation between choroidal scars and active lesions. Int. Ophthalmol. 33, 571-577 (2013).

31. Zheng, $\mathrm{X}$. et al. Indocyanine green-containing nanostructure as near infrared dual-functional targeting probes for optical imaging and photothermal therapy. Mol. Pharm. 8, 447-456 (2011).

32. Kuo, W.-S. et al. Gold Nanomaterials conjugated with indocyanine green for dual-modality photodynamic and photothermal therapy. Biomaterials 33, 3270-3278 (2012)

33. Zheng, X. et al. Enhanced tumor treatment using biofunctional indocyanine green-containing nanostructure by intratumoral or intravenous injection. Mol. Pharm. 9, 514-522 (2012).

34. Zheng, M. et al. Single-step assembly of DOX/ICG loaded lipid-polymer nanoparticles for highly effective chemo-photothermal combination therapy. ACS Nano 7, 2056-2067 (2013).

35. Chen, L. et al. General methodology of using oil-in-water and water-in-oil emulsions for coiling nanofilaments. J. Am. Chem. Soc. 135, 835-843 (2013).

36. Hou, Y. et al. Controlled synthesis and chemical conversions of $\mathrm{FeO}$ nanoparticles. Angew. Chem. Int. Ed. 119, 6445-6448 (2007).

37. Wang, D. et al. Core-shell metal-organic frameworks as $\mathrm{Fe} 2+$ suppliers for Fe2+-mediated cancer therapy under multimodality imaging. Chem. Mater. 29, 3477-3489 (2017)

38. Dixon, S. J. et al. Ferroptosis: an iron-dependent form of nonapoptotic cell death. Cell 149, 1060-1072 (2012).

39. Dolma, S. et al. Identification of genotype-selective antitumor agents using synthetic lethal chemical screening in engineered human tumor cells. Cancer Cell 3, 285-296 (2003).

40. Yagoda, N. et al. RAS-RAF-MEK-dependent oxidative cell death involving voltage-dependent anion channels. Nature 447, 864-868 (2007).

41. Yang, W. S. et al. Synthetic lethal screening identifies compounds activating iron-dependent, nonapoptotic cell death in oncogenic-RAS-harboring cancer cells. Chem. Biol. 15, 234-245 (2008).

42. Kim, S. E. et al. Ultrasmall nanoparticles induce ferroptosis in nutrientdeprived cancer cells and suppress tumour growth. Nat. Nanotechnol. 11, 977-985 (2016).

43. Zanganeh, S. et al. Iron oxide nanoparticles inhibit tumour growth by inducing pro-inflammatory macrophage polarization in tumour tissues. Nat. Nanotechnol. 11, 986-994 (2016).

44. Deepagan, V. G. et al. Long-circulating $\mathrm{Au}-\mathrm{TiO}_{2}$ nanocomposite as a sonosensitizer for ROS-mediated eradication of cancer. Nano Lett. 16 6257-6264 (2016)

45. Chang, K. et al. Enhanced phototherapy by nanoparticle-enzyme via generation and photolysis of hydrogen peroxide. Nano Lett. 17, 4323-4329 (2017)

46. Kosaka, K. et al. Comparison among the methods for hydrogen peroxide measurements to evaluate advanced oxidation processes: application of a spectrophotometric method using copper (II) ion and 2,9-dimethyl-1, 10 phenanthroline. Environ. Sci. Technol. 32, 3821-3824 (1998).

47. Yang, X.-J. et al. Iron oxychloride (FeOCl): an efficient Fenton-like catalyst for producing hydroxyl radicals in degradation of organic contaminants. J. Am. Chem. Soc. 135, 16058-16061 (2013).

48. $\mathrm{Li}, \mathrm{H}$. et al. Numerical analysis of stability for revolutionary thin shell. Acta Mech. Sin. 22, 110-113 (1990).

49. Lee, K. G. et al. In vitro biosynthesis of metal nanoparticles in microdroplets ACS Nano 6, 6998-7008 (2012).

50. Tokarev, I. et al. Stimuli-responsive porous hydrogels at interfaces for molecular filtration, separation, controlled release, and gating in capsules and membranes. Adv. Mater. 22, 3446-3462 (2010)

51. Shih, H. et al. Cross-linking and degradation of step-growth hydrogels formed by thiol-ene photoclick chemistry. Biomacromolecules 13, 2003-2012 (2012).

52. Ulbrich, K. et al. Targeted drug delivery with polymers and magnetic nanoparticles: covalent and noncovalent approaches, release control, and clinical studies. Chem. Rev. 116, 5338-5431 (2016).

53. Kamaly, N. et al. Degradable controlled-release polymers and polymeric nanoparticles: mechanisms of controlling drug release. Chem. Rev. 116, 2602-2663 (2016)

54. Gangloff, N. et al. Peptoids and polypeptoids at the frontier of supra-and macromolecular engineering. Chem. Rev. 116, 1753-1802 (2016).

55. Spicer, C. D. et al. Achieving controlled biomolecule-biomaterial conjugation Chem. Rev. 118, 7702-7743 (2018).

56. Cabral, $\mathrm{H}$. et al. Block copolymer micelles in nanomedicine applications. Chem. Rev. 118, 6844-6892 (2018).

57. Cho, E. C. et al. The effect of sedimentation and diffusion on cellular uptake of gold nanoparticles. Nat. Nanotechnol. 6, 385-391 (2011).

58. Yang, X. et al. Gold nanomaterials at work in biomedicine. Chem. Rev. 115, 10410-10488 (2015) 
59. Dai, Y. et al. Nanoparticle design strategies for enhanced anticancer therapy by exploiting the tumour microenvironment. Chem. Soc. Rev. 46, 3830-3852 (2017).

60. Yang, Z. et al. Modulating the phases of iron carbide nanoparticles: from a perspective of interfering with the carbon penetration of $\mathrm{Fe} @ \mathrm{Fe}_{3} \mathrm{O}_{4}$ by selectively adsorbed halide ions. Chem. Sci. 8, 473-481 (2017).

61. Liu, J. et al. A high-performance imaging probe with NIR luminescence and synergistically enhanced T1-T2 relaxivity for in vivo hepatic tumor targeting and multimodal imaging. Chem. Commun. 51, 13369-13372 (2015).

62. Wang, $\mathrm{Z}$. et al. Controlled synthesis of $\mathrm{MnFe}_{2} \mathrm{O}_{4}$ nanoparticles and $\mathrm{Gd}$ complex-based nanocomposites as tunable and enhanced T1/T2-weighted MRI contrast agents. J. Mater. Chem. B 2, 4748-4753 (2014).

63. Wang, B. et al. Coupling of luminescent terbium complexes to $\mathrm{Fe}_{3} \mathrm{O}_{4}$ nanoparticles for imaging applications. Angew. Chem. Int. Ed. 50, 3063-3066 (2011).

\section{Acknowledgements}

This work was financially supported by the Beijing Municipalit Unicipality of Natural Science Foundation (L72008), the National Natural Science Foundation of China (51672010, 21671088, 81421004, 21431002, 51631001, 51590882, 21575161), the National Key R\&D Program of China (2017YFA0206301, 2016YFA0200102), and the Key Laboratory of Biomedical Effects of Nanomaterials and Nanosafety, National Center for Nanoscience and Technology, Chinese Academy of Sciences (NSKF201607). Thanks for the help of the radial stress analysis for nanocapsules in the shrinking process from Fuyao Zhao.

\section{Author contributions}

B.W. and Y.H. conceived and designed the experiments. Z.W., Y.J., H.Y., F.S. and J.L. performed the experiments. Z.W., B.W. and Y.H. analyzed the results. Z.W., Y.J., Z.A., B.W. and Y.H. wrote the manuscript.

\section{Competing interests}

The authors declare no competing interests.

\section{Additional information}

Supplementary information is available for this paper at https://doi.org/10.1038/s41467019-12142-4.

Correspondence and requests for materials should be addressed to F.S., B.W. or Y.H.

Peer review information Nature Communications thanks the anonymous reviewers for their contributions to the peer review of this work. Peer review reports are available

Reprints and permission information is available at http://www.nature.com/reprints

Publisher's note Springer Nature remains neutral with regard to jurisdictional claims in published maps and institutional affiliations.

(c) (i) Open Access This article is licensed under a Creative Commons Attribution 4.0 International License, which permits use, sharing, adaptation, distribution and reproduction in any medium or format, as long as you give appropriate credit to the original author(s) and the source, provide a link to the Creative Commons license, and indicate if changes were made. The images or other third party material in this article are included in the article's Creative Commons license, unless indicated otherwise in a credit line to the material. If material is not included in the article's Creative Commons license and your intended use is not permitted by statutory regulation or exceeds the permitted use, you will need to obtain permission directly from the copyright holder. To view a copy of this license, visit http://creativecommons.org/ licenses/by/4.0/.

(C) The Author(s) 2019 